MATHEMATICS OF COMPUTATION

Volume 68, Number 226, April 1999, Pages 607-631

S 0025-5718(99)01013-3

\title{
AN OPTIMAL DOMAIN DECOMPOSITION PRECONDITIONER FOR LOW-FREQUENCY TIME-HARMONIC MAXWELL EQUATIONS
}

\author{
ANA ALONSO AND ALBERTO VALLI
}

\begin{abstract}
The time-harmonic Maxwell equations are considered in the lowfrequency case. A finite element domain decomposition approach is proposed for the numerical approximation of the exact solution. This leads to an iteration-by-subdomain procedure, which is proven to converge. The rate of convergence turns out to be independent of the mesh size, showing that the preconditioner implicitly defined by the iterative procedure is optimal. For obtaining this convergence result it has been necessary to prove a regularity theorem for Dirichlet and Neumann harmonic fields.
\end{abstract}

\section{INTRODUCTION}

The Maxwell equations read

$$
\begin{aligned}
& \frac{\partial \mathcal{D}}{\partial t}=\operatorname{rot} \mathcal{H}-\mathcal{J}, \\
& \frac{\partial \mathcal{B}}{\partial t}=-\operatorname{rot} \mathcal{E},
\end{aligned}
$$

where $\mathcal{E}$ and $\mathcal{H}$ are the electric and magnetic field, $\mathcal{D}$ and $\mathcal{B}$ the electric and magnetic induction, respectively, and $\mathcal{J}$ is the density of the electric current. The following constitutive relations

$$
\mathcal{D}=\varepsilon \mathcal{E}, \quad \mathcal{B}=\mu \mathcal{H}
$$

(where $\varepsilon$ and $\mu$ are the dielectric and magnetic permeability coefficients, respectively) are assumed to hold, as well as the Ohm's law

$$
\mathcal{J}=\sigma \mathcal{E}
$$

(where $\sigma$ is the electric conductivity). The quantities $\varepsilon, \mu$ and $\sigma$ are in general symmetric matrices, depending on the space variable $\mathbf{x}$; $\varepsilon$ and $\mu$ are assumed to be positive definite, whereas $\sigma$ is positive definite in a conductor and vanishing in an insulator.

Received by the editor December 2, 1996 and, in revised form, July 30, 1997.

1991 Mathematics Subject Classification. Primary 65N55, 65N30; Secondary 35Q60.

Key words and phrases. Domain decomposition methods, Maxwell equations.

Partially supported by H.C.M. contract CHRX 0930407. 
Writing the Maxwell equations in terms of $\mathcal{E}$ and $\mathcal{H}$ only, we find

$$
\begin{aligned}
\varepsilon \frac{\partial \mathcal{E}}{\partial t} & =\operatorname{rot} \mathcal{H}-\sigma \mathcal{E}, \\
\mu \frac{\partial \mathcal{H}}{\partial t} & =-\operatorname{rot} \mathcal{E} .
\end{aligned}
$$

We are interested in the so-called time-harmonic case, i.e., we assume that $\mathcal{E}$ and $\mathcal{H}$ are given by

$$
\begin{aligned}
\mathcal{E}(t, \mathbf{x}) & =\operatorname{Re}[\mathbf{E}(\mathbf{x}) \exp (i \alpha t)], \\
\mathcal{H}(t, \mathbf{x}) & =\operatorname{Re}[\mathbf{H}(\mathbf{x}) \exp (i \alpha t)],
\end{aligned}
$$

where $\mathbf{E}$ and $\mathbf{H}$ are three-dimensional complex-valued vector fields, and $\alpha \neq 0$ is a given angular frequency. Therefore, the equations become

$$
\begin{aligned}
i \alpha \varepsilon \mathbf{E} & =\operatorname{rot} \mathbf{H}-\sigma \mathbf{E}, \\
i \alpha \mu \mathbf{H} & =-\operatorname{rot} \mathbf{E},
\end{aligned}
$$

and eliminating $\mathbf{H}$ we find

$$
\operatorname{rot}\left(\mu^{-1} \operatorname{rot} \mathbf{E}\right)-\alpha^{2}\left(\varepsilon-i \alpha^{-1} \sigma\right) \mathbf{E}=\mathbf{0} .
$$

If we are considering the low-frequency case, i.e., the parameter $\alpha$ is small, by checking the effective values of the dielectric coefficient $\varepsilon$, the magnetic permeability $\mu$ and the conductivity $\sigma$ for general media, it can be seen that the parameter $\alpha^{2} \varepsilon$ is much smaller than $\mu^{-1}$ and $\alpha \sigma$. Therefore, in this case the term $\alpha^{2} \varepsilon \mathbf{E}$ can be dropped out, and one is left with

$$
\operatorname{rot}\left(\mu^{-1} \operatorname{rot} \mathbf{E}\right)+i \alpha \sigma \mathbf{E}=\mathbf{0}
$$

Formally speaking, the low-frequency model is thus obtained from the general equation (1.2) by setting $\varepsilon=0$. Afterwards we will refer to the low-frequency case as to the case where $\varepsilon=0$.

Considering (1.1) or (1.2) in a bounded domain $\Omega \subset \mathbf{R}^{3}$, we have to impose the boundary condition

$$
\mathbf{n} \times \mathbf{E}=\mathbf{\Psi} \text { on } \partial \Omega,
$$

where $\mathbf{n}$ is the unit outward normal vector on $\partial \Omega$ and $\boldsymbol{\Psi}$ is a tangential vector on $\partial \Omega$.

Most often, it is assumed that a vector function $\widehat{\mathbf{E}}$ is known, satisfying $\mathbf{n} \times \widehat{\mathbf{E}}=\mathbf{\Psi}$ on $\partial \Omega$. Then the resulting boundary value problem reads

$$
\begin{cases}\operatorname{rot}\left(\mu^{-1} \operatorname{rot} \mathbf{u}\right)-\alpha^{2}\left(\varepsilon-i \alpha^{-1} \sigma\right) \mathbf{u}=\mathbf{F} & \text { in } \Omega, \\ (\mathbf{n} \times \mathbf{u})_{\mid \partial \Omega}=\mathbf{0} & \text { on } \partial \Omega,\end{cases}
$$

where $\mathbf{u}=\mathbf{E}-\widehat{\mathbf{E}}$ and $\mathbf{F}=-\operatorname{rot}\left(\mu^{-1} \operatorname{rot} \widehat{\mathbf{E}}\right)+\alpha^{2}\left(\varepsilon-i \alpha^{-1} \sigma\right) \widehat{\mathbf{E}}$.

Let us now make precise some notation. As usual, we indicate by $H^{k}(\Omega), k \geq 0$, the Sobolev space of (classes of equivalence of) real or complex functions belonging to $L^{2}(\Omega)$ together with all their distributional derivatives of order less than or equal to $k$. In particular, $L^{2}(\Omega)=H^{0}(\Omega)$. We also consider the Sobolev space $H^{s}(\Omega)$ for $s \in \mathbf{R}$, whose definition can be found in Adams [1].

It is well known that the trace space of $H^{1}(\Omega)$ over $\partial \Omega$ is given by the Sobolev space $H^{1 / 2}(\partial \Omega$ ); more generally, if $\Sigma$ is a proper (non-empty) subset of $\partial \Omega$, the trace space of $H^{1}(\Omega)$ over $\Sigma$ is given by $H^{1 / 2}(\Sigma)$. The spaces $H^{-1 / 2}(\partial \Omega)$ and 
$H^{-1 / 2}(\Sigma)$ are the dual spaces of $H^{1 / 2}(\partial \Omega)$ and $H^{1 / 2}(\Sigma)$, respectively. The duality pairing between these spaces will be denoted by $\langle\cdot, \cdot\rangle_{\partial \Omega}$ and $\langle\cdot, \cdot\rangle_{\Sigma}$. The norm in the Sobolev space $H^{s}(\Xi)$ will be denoted by $\|\cdot\|_{s, \Xi}$, where $s \in \mathbf{R}$ and $\Xi$ can be either the whole domain $\Omega$, or the boundary $\partial \Omega$, or else a suitable surface.

The space $H(\operatorname{rot} ; \Omega)$ (respectively, $H(\operatorname{div} ; \Omega)$ ) indicates the set of the real or complex (vector) functions $\mathbf{v} \in\left(L^{2}(\Omega)\right)^{3}$ such that $\operatorname{rot} \mathbf{v} \in\left(L^{2}(\Omega)\right)^{3}$ (respectively, $\operatorname{div} \mathbf{v} \in L^{2}(\Omega)$ ).

We also need the definition of the tangential divergence of a tangential vector field $\boldsymbol{\eta}$. Being given $\boldsymbol{\eta} \in\left(H^{-1 / 2}(\partial \Omega)\right)^{3}$ with $(\boldsymbol{\eta} \cdot \mathbf{n})_{\mid \partial \Omega}=0$, we define the tangential divergence $\operatorname{div}_{\tau} \boldsymbol{\eta}$ of $\boldsymbol{\eta}$ as the distribution in $H^{-3 / 2}(\partial \Omega)$ which satisfies

$$
\left\langle\left\langle\operatorname{div}_{\tau} \boldsymbol{\eta}, \psi\right\rangle\right\rangle_{\partial \Omega}:=-\left\langle\boldsymbol{\eta},\left(\nabla \psi_{2}^{*}\right)_{\mid \partial \Omega}\right\rangle_{\partial \Omega} \quad \forall \psi \in H^{3 / 2}(\partial \Omega),
$$

where $\psi_{2}^{*} \in H^{2}(\Omega)$ is any extension of $\psi$ in $\Omega$, and we have denoted by $\langle\langle\cdot, \cdot\rangle\rangle_{\partial \Omega}$ the duality pairing between $H^{-3 / 2}(\partial \Omega)$ and $H^{3 / 2}(\partial \Omega)$. Notice that, due to the condition $(\boldsymbol{\eta} \cdot \mathbf{n})_{\mid \partial \Omega}=0$, the right hand side indeed depends only on the value of $\psi$ on $\partial \Omega$.

We can now introduce the Hilbert spaces $\mathcal{X}_{\partial \Omega}$ and $\mathcal{X}_{\Sigma}$, where $\Sigma$ is a proper (non-empty) subset of $\partial \Omega$. The former one is defined as

$$
\mathcal{X}_{\partial \Omega}:=\left\{\boldsymbol{\eta} \in\left(H^{-1 / 2}(\partial \Omega)\right)^{3} \mid(\boldsymbol{\eta} \cdot \mathbf{n})_{\mid \partial \Omega}=0 \text { and } \operatorname{div}_{\tau} \boldsymbol{\eta} \in H^{-1 / 2}(\partial \Omega)\right\},
$$

with the norm

$$
\left\|\left.\boldsymbol{\eta}\right|_{\mathcal{X}_{\partial \Omega}}:=\right\| \boldsymbol{\eta}\left\|_{-1 / 2, \partial \Omega}+\right\| \operatorname{div}_{\tau} \boldsymbol{\eta} \|_{-1 / 2, \partial \Omega}
$$

Denoting by $\widetilde{\gamma} \in\left(H^{-1 / 2}(\partial \Omega)\right)^{3}$ the extension of $\boldsymbol{\gamma}$ by $\mathbf{0}$ on $\partial \Omega \backslash \Sigma$, the space $\mathcal{X}_{\Sigma}$ is

$$
\mathcal{X}_{\Sigma}:=\left\{\boldsymbol{\gamma} \in\left(H^{-1 / 2}(\Sigma)\right)^{3} \mid(\boldsymbol{\gamma} \cdot \mathbf{n})_{\mid \Sigma}=0 \text { and } \operatorname{div}_{\tau} \widetilde{\gamma} \in H^{-1 / 2}(\partial \Omega)\right\},
$$

endowed with the norm

$$
\|\gamma\|_{\mathcal{X}_{\Sigma}}:=\|\gamma\|_{-1 / 2, \Sigma}+\left\|\operatorname{div}_{\tau} \widetilde{\gamma}\right\|_{-1 / 2, \partial \Omega}
$$

In Alonso and Valli [2] it has been proven that, if either $\partial \Omega \in C^{1,1}$ or $\Omega$ is a convex polyhedron, the space $\mathcal{X}_{\partial \Omega}$ is equal, algebraically and topologically, to the space of tangential traces of $H(\operatorname{rot} ; \Omega)$. Similarly, $\mathcal{X}_{\Sigma}$ is the space of tangential traces of

$$
H_{\partial \Omega \backslash \Sigma}(\operatorname{rot} ; \Omega):=\left\{\mathbf{v} \in H(\operatorname{rot} ; \Omega) \mid(\mathbf{n} \times \mathbf{v})_{\mid \partial \Omega \backslash \Sigma}=\mathbf{0}\right\}
$$

Furthermore, in [2] it has been shown that there exist two linear and continuous extension operators

$$
\begin{aligned}
\mathcal{R}_{\partial \Omega} & : \mathcal{X}_{\partial \Omega} \rightarrow H(\operatorname{rot} ; \Omega), \\
\mathcal{R}_{\Sigma}: & \mathcal{X}_{\Sigma} \rightarrow H_{\partial \Omega \backslash \Sigma}(\operatorname{rot} ; \Omega)
\end{aligned}
$$

satisfying

$$
\left(\mathbf{n} \times \mathcal{R}_{\partial \Omega} \boldsymbol{\eta}\right)_{\mid \partial \Omega}=\boldsymbol{\eta}, \quad\left(\mathbf{n} \times \mathcal{R}_{\Sigma} \boldsymbol{\gamma}\right)_{\mid \Sigma}=\boldsymbol{\gamma}
$$

for each $\boldsymbol{\eta} \in \mathcal{X}_{\partial \Omega}$ and $\boldsymbol{\gamma} \in \mathcal{X}_{\Sigma}$. 


\section{WEAK FORMULATION OF THE PROBLEM AND FINITE ELEMENT APPROXIMATION}

We are going to make precise the variational formulation. First of all, we need the following notation:

$$
\begin{aligned}
& H_{0}(\operatorname{rot} ; \Omega):=\left\{\mathbf{v} \in H(\operatorname{rot} ; \Omega) \mid(\mathbf{n} \times \mathbf{v})_{\mid \partial \Omega}=\mathbf{0}\right\} \\
& H^{0}(\operatorname{rot} ; \Omega):=\{\mathbf{v} \in H(\operatorname{rot} ; \Omega) \mid \operatorname{rot} \mathbf{v}=\mathbf{0}\} \\
& H_{0}(\operatorname{div} ; \Omega):=\left\{\mathbf{v} \in H(\operatorname{div} ; \Omega) \mid(\mathbf{n} \cdot \mathbf{v})_{\mid \partial \Omega}=0\right\} \\
& H^{0}(\operatorname{div} ; \Omega):=\{\mathbf{v} \in H(\operatorname{div} ; \Omega) \mid \operatorname{div} \mathbf{v}=0\}
\end{aligned}
$$

We also assume that the coefficients $\mu=\left(\mu_{i j}(\mathbf{x})\right)_{1 \leq i, j \leq 3}, \varepsilon=\left(\varepsilon_{i j}(\mathbf{x})\right)_{1 \leq i, j \leq 3}$ and $\sigma=\left(\sigma_{i j}(\mathbf{x})\right)_{1 \leq i, j \leq 3}$ are symmetric matrices with real coefficients belonging to $L^{\infty}(\Omega)$. The magnetic permeability is uniformly positive definite (UPD from now on); namely, there exists a constant $\mu_{0}>0$ such that

$$
\sum_{l, m=1}^{3} \mu_{l m}(\mathbf{x}) \xi_{l} \bar{\xi}_{m} \geq \mu_{0}|\boldsymbol{\xi}|^{2} \quad \text { for almost all } x \in \Omega \text { and for all } \boldsymbol{\xi} \in \mathbf{C}^{3} .
$$

The dielectric coefficient $\varepsilon$ is assumed to be UPD in the high-frequency case and 0 in the low-frequency case. The conductivity $\sigma$ can be UPD (when $\Omega$ is a conductor), or else given by $\sigma=\widehat{\sigma} \chi_{\Omega \backslash \Omega_{0}}$, where $\Omega_{0}$ is a (non-empty) subset of $\Omega$ (representing an insulator), $\chi_{\Omega \backslash \Omega_{0}}$ is the characteristic function of $\Omega \backslash \Omega_{0}$, and $\widehat{\sigma}$ is UPD. In particular, the case $\Omega_{0}=\Omega$ corresponds to the case of a perfect insulator.

We introduce in $H(\operatorname{rot} ; \Omega)$ the following bilinear form:

$$
a_{\varepsilon}(\mathbf{w}, \mathbf{v}):=\left(\mu^{-1} \operatorname{rot} \mathbf{w}, \operatorname{rot} \mathbf{v}\right)-\alpha^{2}\left(\left[\varepsilon-i \alpha^{-1} \sigma\right] \mathbf{w}, \mathbf{v}\right),
$$

where $(\cdot, \cdot)$ denotes the $\left(L^{2}(\Omega)\right)^{3}$-scalar product (for complex-valued vector functions), and we set $L(\mathbf{v}):=(\mathbf{F}, \mathbf{v})$.

Definition 2.1. A weak solution of (1.5) is a function $\mathbf{u} \in H_{0}(\operatorname{rot} ; \Omega)$ such that

$$
a_{\varepsilon}(\mathbf{u}, \mathbf{v})=L(\mathbf{v}) \quad \forall \mathbf{v} \in H_{0}(\operatorname{rot} ; \Omega) .
$$

The high-frequency case ( $\varepsilon$ is assumed to be UPD) has been considered by Leis [9]. First of all, the bilinear form $a_{\varepsilon}(\cdot, \cdot)$ has been proven to be coercive in $H(\operatorname{rot} ; \Omega)$ when $\sigma$ is UPD. Moreover, the Fredholm alternative theorem holds for problem (2.1) when $\sigma=0$ (i.e., $\Omega_{0}=\Omega$ ) (see [9]). A unique solvability result has been proven by Alonso and Valli [4] for the conductivity given by $\sigma=\widehat{\sigma} \chi_{\Omega \backslash \Omega_{0}}, \Omega_{0} \neq \Omega$.

We are mainly interested in the sequel in the low-frequency case $(\varepsilon$ is taken to be 0 ) for a conductor ( $\sigma$ is assumed to be UPD). In that case we can verify at once that the bilinear form $a_{0}(\cdot, \cdot)$ is continuous and coercive in $H(\operatorname{rot} ; \Omega)$; therefore, the Lax-Milgram lemma yields

Theorem 2.2. Let $\Omega$ be a bounded domain, and assume that $\varepsilon=0$ and $\sigma$ is UPD. Then there exists a unique solution of (2.1).

A different approach is needed in the low-frequency case when the conductivity is given by $\sigma=\widehat{\sigma} \chi_{\Omega \backslash \Omega_{0}}$. In this case the problem

$$
\begin{cases}\operatorname{rot}\left(\mu^{-1} \operatorname{rot} \mathbf{u}\right)+i \alpha \sigma \mathbf{u}=\mathbf{F} & \text { in } \Omega, \\ (\mathbf{n} \times \mathbf{u})_{\mid \partial \Omega}=\mathbf{0} & \text { on } \partial \Omega,\end{cases}
$$


does not have a unique solution, as we can always add the gradient of a harmonic function supported in $\Omega_{0}$ to a solution. Therefore, we have to complete the differential model by adding suitable equations.

Alonso and Valli [3], by means of a perturbation argument, have proposed the following problem:

$$
\begin{cases}\operatorname{rot}\left(\mu^{-1} \operatorname{rot} \mathbf{u}\right)+i \alpha \sigma \mathbf{u}=\mathbf{F} & \text { in } \Omega, \\ \operatorname{div}\left(\mathbf{u}_{\mid \Omega_{0}}\right)=0 & \text { in } \Omega_{0}, \\ (\mathbf{n} \times \mathbf{u})_{\mid \partial \Omega}=\mathbf{0} & \text { on } \partial \Omega, \\ \left\langle\left(\mathbf{u}_{\mid \Omega_{0}} \cdot \mathbf{n}\right)_{\mid \Gamma_{0, j}}, 1\right\rangle_{\Gamma_{0, j}}=0 & \forall j=1, \ldots, p,\end{cases}
$$

where $\Gamma_{0, j}$ are the internal connected components of $\partial \Omega_{0}$. In [3] it has been proven that (2.3) has a unique solution when $\Omega_{0} \neq \Omega$, and the interface $\partial \Omega_{0} \cap \partial\left(\Omega \backslash \Omega_{0}\right)$ is either a $C^{1,1}$ surface or a convex polyhedral portion of $\partial \Omega_{0}$.

Finally, in the case $\Omega_{0}=\Omega$ (i.e., $\sigma=0$ ) problem (2.3) reduces to a coercive problem in $H_{0}(\operatorname{rot} ; \Omega) \cap H(\operatorname{div} ; \Omega) \cap \mathcal{H}(e)^{\perp}$, having set

$$
\mathcal{H}(e):=\left\{\boldsymbol{\omega} \in H^{0}(\operatorname{rot} ; \Omega) \cap H^{0}(\operatorname{div} ; \Omega) \mid(\mathbf{n} \times \boldsymbol{\omega})_{\mid \partial \Omega}=\mathbf{0}\right\}
$$

(see Saranen [15], Valli [17]).

We are now going to present some approximation results that have been obtained for problem (2.1) via the finite element method.

In the low-frequency case for a conductor, the bilinear form $a_{0}(\cdot, \cdot)$ is coercive in $H(\operatorname{rot} ; \Omega)$; therefore, the problem is rather standard, and one only needs to construct a suitable internal finite dimensional approximation of the space $H(\operatorname{rot} ; \Omega)$. To this end, the so-called Nédélec finite elements (see Nédélec [12], [13]) can be used, as they are conforming in $H(\operatorname{rot} ; \Omega)$ (their tangential components are continuous across the faces of the finite elements). An optimal order error estimate can be obtained straightforwardly.

In the same case, by means of a different approach, Kř́ížek and Neittaanmäki [8] proposed a finite element space given by standard Lagrangian piecewise-linear vector functions satisfying suitable conditions on the interfaces. In particular, when $\sigma$ is a constant, these conditions reduce to the continuity across the interfaces, thus furnishing a finite dimensional subspace of $\left(H^{1}(\Omega)\right)^{3}$.

Also in the high-frequency case Monk [10] has used the Nédélec finite elements, both for the case where $\sigma$ is assumed to be UPD and for $\sigma=0$, yielding an optimal order error estimate.

The low-frequency heterogeneous problem (2.3), in which $\sigma=\widehat{\sigma} \chi_{\Omega \backslash \Omega_{0}}, \Omega_{0} \neq \Omega$, has been considered in [3]. At first the problem has been rewritten in an equivalent two-domain formulation, and then the Nédélec finite elements are employed in $\Omega \backslash \Omega_{0}$, whereas Lagrangian piecewise-polynomial finite elements are used in $\Omega_{0}$ for approximating a scalar potential of the magnetic field.

Due to the heterogeneous nature of the problem, a natural domain decomposition algorithm can be devised, solving the problem iteratively in $\Omega_{0}$ and in $\Omega \backslash \Omega_{0}$. The convergence of this iterative procedure is proven in [3], where the rate of convergence is also shown to be independent of the mesh size $h$.

Clearly, it is also interesting to use a domain decomposition technique for solving the two subproblems in $\Omega_{0}$ and in $\Omega \backslash \Omega_{0}$. For what is concerned with the problem in 
the perfect insulator $\Omega_{0}$, is equivalent to the construction of an extension operator from $\mathcal{X}_{\partial \Omega_{0}}$ into $H\left(\operatorname{rot} ; \Omega_{0}\right)$ (see Alonso and Valli [2]). Moreover, this last problem can be reduced to a non-homogeneous Neumann boundary value problem for the Laplace operator, and domain decomposition techniques for its finite element approximation are well known.

In the next section we are going to consider the domain decomposition approach to the finite element approximation of the low-frequency conductor problem, namely the case where it is assumed that $\varepsilon=0$ and that $\sigma$ is UPD.

\section{The domain DeComposition PRocedure}

We consider the low-frequency conductor problem

$$
\begin{cases}\operatorname{rot}\left(\mu^{-1} \operatorname{rot} \mathbf{u}\right)+i \alpha \sigma \mathbf{u}=\mathbf{F} & \text { in } \Omega, \\ (\mathbf{n} \times \mathbf{u})_{\mid \partial \Omega}=\mathbf{0} & \text { on } \partial \Omega .\end{cases}
$$

The bilinear form associated to (3.1) is given by

$$
a_{0}(\mathbf{w}, \mathbf{v}):=\int_{\Omega}\left(\mu^{-1} \operatorname{rot} \mathbf{w} \cdot \operatorname{rot} \overline{\mathbf{v}}+i \alpha \sigma \mathbf{w} \cdot \overline{\mathbf{v}}\right),
$$

and the weak formulation reads as in Definition 2.1.

Let the bounded domain $\Omega$ be decomposed in two subdomains $\Omega_{1}$ and $\Omega_{2}$ such that $\bar{\Omega}=\overline{\Omega_{1}} \cup \overline{\Omega_{2}}$ and $\Omega_{1} \cap \Omega_{2}=\emptyset$. We will set $\Gamma:=\overline{\Omega_{1}} \cap \overline{\Omega_{2}}$.

In each subdomain we want to solve

$$
\begin{cases}\operatorname{rot}\left(\mu^{-1} \operatorname{rot} \mathbf{u}_{j}\right)+i \alpha \sigma \mathbf{u}_{j}=\mathbf{F} & \text { in } \Omega_{j}, \\ \left(\mathbf{n} \times \mathbf{u}_{j}\right)_{\mid \partial \Omega_{j} \backslash \Gamma}=\mathbf{0} & \text { on } \partial \Omega_{j} \backslash \Gamma\end{cases}
$$

with the interface conditions

Set

$$
\begin{aligned}
\left(\mathbf{n}_{\Gamma} \times \mathbf{u}_{1}\right)_{\mid \Gamma} & =\left(\mathbf{n}_{\Gamma} \times \mathbf{u}_{2}\right)_{\mid \Gamma}, \\
\left(\mathbf{n}_{\Gamma} \times \mu^{-1} \operatorname{rot} \mathbf{u}_{1}\right)_{\mid \Gamma} & =\left(\mathbf{n}_{\Gamma} \times \mu^{-1} \operatorname{rot} \mathbf{u}_{2}\right)_{\mid \Gamma} .
\end{aligned}
$$

$$
\begin{gathered}
V_{j}:=\left\{\mathbf{v}_{j} \in H\left(\operatorname{rot} ; \Omega_{j}\right) \mid\left(\mathbf{n} \times \mathbf{v}_{j}\right)_{\mid \partial \Omega_{j} \backslash \Gamma}=\mathbf{0}\right\} \\
a_{j}\left(\mathbf{w}_{j}, \mathbf{v}_{j}\right):=\int_{\Omega_{j}}\left(\mu^{-1} \operatorname{rot} \mathbf{w}_{j} \cdot \operatorname{rot} \overline{\mathbf{v}_{j}}+i \alpha \sigma \mathbf{w}_{j} \cdot \overline{\mathbf{v}_{j}}\right), \quad \forall \mathbf{w}_{j}, \mathbf{v}_{j} \in V_{j}, \\
L_{j}\left(\mathbf{v}_{j}\right):=\int_{\Omega_{j}} \mathbf{F} \cdot \overline{\mathbf{v}_{j}} .
\end{gathered}
$$


The bilinear forms $a_{j}$ are clearly continuous and coercive in $V_{j}$. The variational formulation of the two-domain problem reads

$$
\left\{\begin{array}{l}
\text { find, }\left(\mathbf{u}_{1}, \mathbf{u}_{2}\right) \in V_{1} \times V_{2}: \\
a_{1}\left(\mathbf{u}_{1}, \mathbf{v}_{1}\right)=L_{1}\left(\mathbf{v}_{1}\right) \quad \forall \mathbf{v}_{1} \in H_{0}\left(\operatorname{rot} ; \Omega_{1}\right) \\
\left(\mathbf{n}_{\Gamma} \times \mathbf{u}_{1}\right)_{\mid \Gamma}=\left(\mathbf{n}_{\Gamma} \times \mathbf{u}_{2}\right)_{\mid \Gamma} \\
a_{2}\left(\mathbf{u}_{2}, \mathbf{v}_{2}\right)=L_{2}\left(\mathbf{v}_{2}\right)+L_{1}\left(\mathbf{R}_{1}\left(\mathbf{n}_{\Gamma} \times \mathbf{v}_{2}\right)_{\mid \Gamma}\right) \\
-a_{1}\left(\mathbf{u}_{1}, \mathbf{R}_{1}\left(\mathbf{n}_{\Gamma} \times \mathbf{v}_{2}\right)_{\mid \Gamma}\right) \quad \forall \mathbf{v}_{2} \in V_{2},
\end{array}\right.
$$

where $\mathbf{R}_{1}: \mathcal{X}_{\Gamma} \rightarrow V_{1}$ is any extension operator.

The equivalence of the formulations (2.1) and (3.8) can be easily proven (see, for instance, Alonso and Valli [3], where a similar situation is considered).

For the numerical approximation, we will use the Nédélec finite elements of the first kind (see Nédélec [12]). However, the same results could be proven also for the Nédélec finite elements of the second kind (see Nédélec [13]). For the reader's convenience, we present here the precise definitions of the former elements.

Let us assume that $\Omega, \Omega_{1}$ and $\Omega_{2}$ are a Lipschitz polyhedrons. Let $\left\{\mathcal{T}_{h}\right\}_{h>0}$ be a family of triangulations composed by tetrahedrons, where $h$ is their maximum diameter. Moreover, assume that each element of $\mathcal{T}_{h}$ only intersects either $\Omega_{1}$ or $\Omega_{2}$. Let $\mathbf{P}_{k}, k \geq 1$, be the space of polynomials of degree less than or equal to $k$, and denote by $\mathbf{P}_{k}^{*}$ the space of homogeneous polynomials of degree $k$. We set

$$
S_{k}:=\left\{\mathbf{p} \in\left(\mathbf{P}_{k}^{*}\right)^{3} \mid \mathbf{p}(\mathbf{x}) \cdot \mathbf{x}=0\right\}, \quad R_{k}:=\left(\mathbf{P}_{k-1}\right)^{3} \oplus S_{k} .
$$

Notice that $\left(\mathbf{P}_{k-1}\right)^{3} \subset R_{k} \subset\left(\mathbf{P}_{k}\right)^{3}$. We will employ the finite element space

$$
N_{j, h}^{k}:=\left\{\mathbf{v}_{h} \in H\left(\operatorname{rot} ; \Omega_{j}\right) \mid \mathbf{v}_{h \mid K} \in R_{k} \forall K \in \mathcal{T}_{j, h}\right\}
$$

and we define

$$
\begin{gathered}
V_{j, h}:=N_{j, h}^{k} \cap V_{j}, \\
V_{j, h}^{0}:=N_{j, h}^{k} \cap H_{0}\left(\operatorname{rot} ; \Omega_{j}\right), \\
\mathcal{X}_{\Gamma, h}:=\left\{\left(\mathbf{n}_{\Gamma} \times \mathbf{v}_{1, h}\right)_{\mid \Gamma} \mid \mathbf{v}_{1, h} \in V_{1, h}\right\}=\left\{\left(\mathbf{n}_{\Gamma} \times \mathbf{v}_{2, h}\right)_{\mid \Gamma} \mid \mathbf{v}_{2, h} \in V_{2, h}\right\} .
\end{gathered}
$$

The finite dimensional approximation problem reads

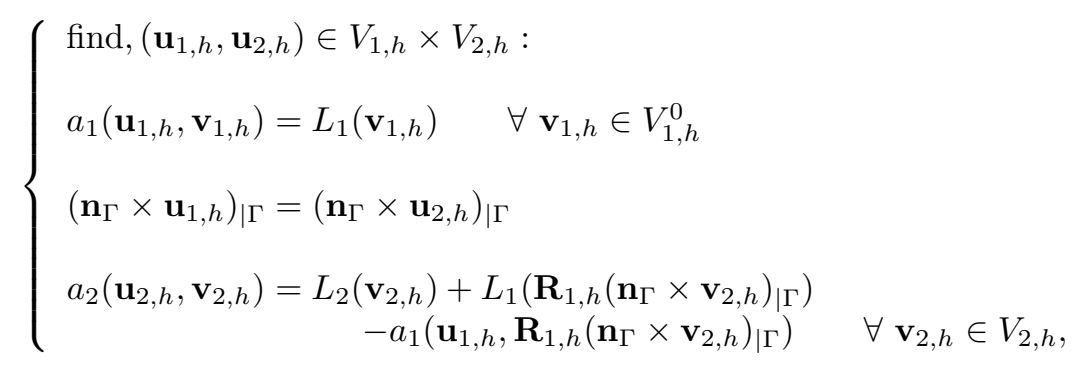

where $\mathbf{R}_{1, h}$ is any extension operator from $\mathcal{X}_{h, \Gamma}$ to $V_{1, h}$. 
Let us introduce now for each $\gamma_{h} \in \mathcal{X}_{h, \Gamma}$ the solution $\mathbf{E}_{j, \Gamma}^{h} \gamma_{h}$ of the problem

$$
\left\{\begin{array}{l}
\mathbf{E}_{j, \Gamma}^{h} \gamma_{h} \in V_{j, h}: \\
a_{j}\left(\mathbf{E}_{j, \Gamma}^{h} \gamma_{h}, \mathbf{v}_{j, h}\right)=0 \quad \forall \mathbf{v}_{j, h} \in V_{j, h}^{0} \\
\left(\mathbf{n}_{\Gamma} \times \mathbf{E}_{j, \Gamma}^{h} \gamma_{h}\right)_{\mid \Gamma}=\gamma_{h},
\end{array}\right.
$$

and also the solution $\widehat{\mathbf{u}}_{j, h} \in V_{j, h}^{0}$ of

$$
\widehat{\mathbf{u}}_{j, h} \in V_{j, h}^{0}: a_{j}\left(\widehat{\mathbf{u}}_{j, h}, \mathbf{v}_{j, h}\right)=L_{j}\left(\mathbf{v}_{j, h}\right) \quad \forall \mathbf{v}_{j, h} \in V_{j, h}^{0},
$$

whose existence and uniqueness is a consequence of Lax-Milgram Lemma. Then the couple $\left(\mathbf{E}_{1, \Gamma}^{h} \boldsymbol{\lambda}_{h}+\widehat{\mathbf{u}}_{1, h}, \mathbf{E}_{2, \Gamma}^{h} \boldsymbol{\lambda}_{h}+\widehat{\mathbf{u}}_{2, h}\right)$ is a solution to (3.12) if and only if

$$
\begin{aligned}
& a_{2}\left(\mathbf{E}_{2, \Gamma}^{h} \boldsymbol{\lambda}_{h}+\widehat{\mathbf{u}}_{2, h}, \mathbf{v}_{2, h}\right)=L_{2}\left(\mathbf{v}_{2, h}\right)+L_{1}\left(\mathbf{E}_{1, \Gamma}^{h}\left(\mathbf{n}_{\Gamma} \times \mathbf{v}_{2, h}\right)_{\mid \Gamma}\right) \\
& \quad-a_{1}\left(\mathbf{E}_{1, \Gamma}^{h} \boldsymbol{\lambda}_{h}+\widehat{\mathbf{u}}_{1, h}, \mathbf{E}_{1, \Gamma}^{h}\left(\mathbf{n}_{\Gamma} \times \mathbf{v}_{2, h}\right)_{\mid \Gamma}\right) \quad \forall \mathbf{v}_{2, h} \in V_{2, h}
\end{aligned}
$$

is satisfied. Due to (3.13) and (3.14), this is equivalent to

$$
\begin{array}{r}
a_{2}\left(\mathbf{E}_{2, \Gamma}^{h} \boldsymbol{\lambda}_{h}, \mathbf{E}_{2, \Gamma}^{h} \boldsymbol{\eta}_{h}\right)+a_{2}\left(\widehat{\mathbf{u}}_{2, h}, \mathbf{E}_{2, \Gamma}^{h} \boldsymbol{\eta}_{h}\right)=L_{2}\left(\mathbf{E}_{2, \Gamma}^{h} \boldsymbol{\eta}_{h}\right)+L_{1}\left(\mathbf{E}_{1, \Gamma}^{h} \boldsymbol{\eta}_{h}\right) \\
-a_{1}\left(\mathbf{E}_{1, \Gamma}^{h} \boldsymbol{\lambda}_{h}, \mathbf{E}_{1, \Gamma}^{h} \boldsymbol{\eta}_{h}\right)-a_{1}\left(\widehat{\mathbf{u}}_{1, h}, \mathbf{E}_{1, \Gamma}^{h} \boldsymbol{\eta}_{h}\right) \quad \forall \boldsymbol{\eta}_{h} \in \mathcal{X}_{\Gamma, h} .
\end{array}
$$

We define the Steklov-Poincaré operators $\mathcal{S}_{j, h}, j=1,2$, in the following way:

$$
\left\langle\left\langle\mathcal{S}_{j, h} \boldsymbol{\gamma}_{h}, \boldsymbol{\eta}_{h}\right\rangle\right\rangle_{h}:=a_{j}\left(\mathbf{E}_{j, \Gamma}^{h} \boldsymbol{\gamma}_{h}, \mathbf{E}_{j, \Gamma}^{h} \boldsymbol{\eta}_{h}\right) \quad \forall \boldsymbol{\gamma}_{h}, \boldsymbol{\eta}_{h} \in \mathcal{X}_{\Gamma, h},
$$

where $\langle\langle\cdot, \cdot\rangle\rangle_{h}$ denotes the duality pairing between $\left(\mathcal{X}_{\Gamma, h}\right)^{\prime}$ and $\mathcal{X}_{\Gamma, h}$.

Define moreover

$$
\begin{aligned}
\left\langle\left\langle\boldsymbol{\Phi}_{h}, \boldsymbol{\eta}_{h}\right\rangle\right\rangle_{h}:=L_{1}\left(\mathbf{E}_{1, \Gamma}^{h} \boldsymbol{\eta}_{h}\right) & -a_{1}\left(\widehat{\mathbf{u}}_{1, h}, \mathbf{E}_{1, \Gamma}^{h} \boldsymbol{\eta}_{h}\right) \\
& +L_{2}\left(\mathbf{E}_{2, \Gamma}^{h} \boldsymbol{\eta}_{h}\right)-a_{2}\left(\widehat{\mathbf{u}}_{2, h}, \mathbf{E}_{2, \Gamma}^{h} \boldsymbol{\eta}_{h}\right) \quad \forall \boldsymbol{\eta}_{h} \in \mathcal{X}_{\Gamma, h} .
\end{aligned}
$$

Problem (3.12) is therefore reduced to finding

$$
\boldsymbol{\lambda}_{h} \in \mathcal{X}_{\Gamma, h}:\left\langle\left\langle\left(\mathcal{S}_{1, h}+\mathcal{S}_{2, h}\right) \boldsymbol{\lambda}_{h}, \boldsymbol{\eta}_{h}\right\rangle\right\rangle_{h}=\left\langle\left\langle\boldsymbol{\Phi}_{h}, \boldsymbol{\eta}_{h}\right\rangle\right\rangle_{h} \quad \forall \boldsymbol{\eta}_{h} \in \mathcal{X}_{\Gamma, h} .
$$

We will see that the operators $\mathcal{S}_{j, h}$ are continuous and coercive in $\mathcal{X}_{\Gamma, h}$; hence, for solving (3.18) we can apply the Richardson method with one of these operators (say, $\mathcal{S}_{2, h}$ ) as a preconditioner.

In other words, given $\boldsymbol{\lambda}_{h}^{0} \in \mathcal{X}_{\Gamma, h}$, for each $m \geq 0$ solve

$$
\begin{aligned}
\boldsymbol{\lambda}_{h}^{m+1} & =\boldsymbol{\lambda}_{h}^{m}+\theta \mathcal{S}_{2, h}^{-1}\left[\boldsymbol{\Phi}_{h}-\left(\mathcal{S}_{1, h}+\mathcal{S}_{2, h}\right) \boldsymbol{\lambda}_{h}^{m}\right] \\
& =(1-\theta) \boldsymbol{\lambda}_{h}^{m}+\theta \mathcal{S}_{2, h}^{-1}\left(\boldsymbol{\Phi}_{h}-\mathcal{S}_{1, h} \boldsymbol{\lambda}_{h}^{m}\right) .
\end{aligned}
$$

By proceeding in a standard way (see, for instance, Alonso and Valli [3], Section 5, for a similar computation), it can be seen that (3.19) is equivalent to the following iteration-by-subdomain algorithm: being given $\boldsymbol{\lambda}_{h}^{0} \in \mathcal{X}_{\Gamma, h}$, for each $m \geq 0$ solve

$$
\left\{\begin{array}{l}
\mathbf{u}_{1, h}^{m+1} \in V_{1, h}: \\
a_{1}\left(\mathbf{u}_{1, h}^{m+1}, \mathbf{v}_{1, h}\right)=L_{1}\left(\mathbf{v}_{1, h}\right) \quad \forall \mathbf{v}_{1, h} \in V_{1, h}^{0} \\
\left(\mathbf{n}_{\Gamma} \times \mathbf{u}_{1, h}^{m+1}\right)_{\mid \Gamma}=\lambda_{h}^{m},
\end{array}\right.
$$




$$
\begin{aligned}
\mathbf{u}_{2, h}^{m+1} \in V_{2, h}: a_{2}\left(\mathbf{u}_{2, h}^{m+1}, \mathbf{v}_{2, h}\right) & =L_{2}\left(\mathbf{v}_{2, h}\right)+L_{1}\left(\mathbf{E}_{1, \Gamma}^{h}\left(\mathbf{n}_{\Gamma} \times \mathbf{v}_{2, h}\right)_{\mid \Gamma}\right) \\
& -a_{1}\left(\mathbf{u}_{1, h}^{m+1}, \mathbf{E}_{1, \Gamma}^{h}\left(\mathbf{n}_{\Gamma} \times \mathbf{v}_{2, h}\right)_{\mid \Gamma}\right) \quad \forall \mathbf{v}_{2, h} \in V_{2, h},
\end{aligned}
$$

and pose

$$
\boldsymbol{\lambda}_{h}^{m+1}:=(1-\theta) \boldsymbol{\lambda}_{h}^{m}+\theta\left(\mathbf{n}_{\Gamma} \times \mathbf{u}_{2, h}^{m+1}\right)_{\mid \Gamma} .
$$

The convergence of the sequence $\boldsymbol{\lambda}_{h}^{m}$ constructed in (3.19) is a consequence of the following abstract theorem.

Theorem 3.1. Let $X$ be a complex Hilbert space and let $\mathcal{S}_{1, h}$ and $\mathcal{S}_{2, h}$ be two linear operators from a finite dimensional space $X_{h} \subset X$ into its dual $X_{h}^{\prime}$. Let $\chi_{s}$, $s=1, \ldots, M_{h}$, a basis of $X_{h}$. Define the matrices $S_{j, h}$ associated to the operators $\mathcal{S}_{j, h}$ as

$$
\left(S_{j, h} \boldsymbol{\gamma}, \boldsymbol{\eta}\right)_{h}:=\left\langle\left\langle\mathcal{S}_{j, h} \gamma_{h}, \boldsymbol{\eta}_{h}\right\rangle\right\rangle_{h} \quad \forall \boldsymbol{\gamma}, \boldsymbol{\eta} \in \mathbf{C}^{M_{h}}, j=1,2,
$$

where $(\cdot, \cdot)_{h}$ denotes the euclidean scalar product in $\mathbf{C}^{M_{h}}$ and

$$
\gamma_{h}:=\sum_{s=1}^{M_{h}} \gamma_{s} \chi_{s}, \quad \boldsymbol{\eta}_{h}:=\sum_{s=1}^{M_{h}} \eta_{s} \chi_{s}
$$

Let us assume that there exist two constants $C_{1}>0$ and $C_{2}>0$, independent of $h$, such that

$$
\begin{gathered}
\left|\left\langle\left\langle\mathcal{S}_{1, h} \gamma_{h}, \boldsymbol{\eta}_{h}\right\rangle\right\rangle_{h}\right| \leq C_{1}\left\|\gamma_{h} \mid\right\|_{X}\left\|\boldsymbol{\eta}_{h}\right\|_{X} \quad \forall \gamma_{h}, \boldsymbol{\eta}_{h} \in X_{h}, \\
\left|\left\langle\left\langle\mathcal{S}_{2, h} \gamma_{h}, \gamma_{h}\right\rangle\right\rangle_{h}\right| \geq C_{2}\left\|\gamma_{h}\right\|_{X}^{2} \quad \forall \gamma_{h} \in X_{h}, \\
\operatorname{Re}\left\langle\left\langle\mathcal{S}_{1, h} \gamma_{h}, \gamma_{h}\right\rangle\right\rangle_{h} \operatorname{Re}\left\langle\left\langle\mathcal{S}_{2, h} \gamma_{h}, \gamma_{h}\right\rangle\right\rangle_{h} \\
+\operatorname{Im}\left\langle\left\langle\mathcal{S}_{1, h} \gamma_{h}, \gamma_{h}\right\rangle\right\rangle_{h}, \operatorname{Im}\left\langle\left\langle\mathcal{S}_{2, h} \gamma_{h}, \gamma_{h}\right\rangle\right\rangle_{h} \geq 0 \quad \forall \gamma_{h} \in X_{h} .
\end{gathered}
$$

Then each eigenvalue $\nu_{s}$ of $S_{2, h}^{-1}\left(S_{1, h}+S_{2, h}\right)$ satisfies

$$
C^{*} \leq 2 \frac{\operatorname{Re} \nu_{s}}{\left|\nu_{s}\right|^{2}} \quad \forall s=1, \ldots, M_{h}
$$

where

$$
C^{*}:=\min \left(1, \frac{2 C_{2}^{2}}{C_{1}^{2}+C_{2}^{2}}\right)
$$

Therefore, for any $\theta \in\left(0, C^{*}\right)$ one has

$$
\left|\nu_{s}\right|^{2}<\frac{2}{\theta} \operatorname{Re} \nu_{s} \quad \forall s=1, \ldots, M_{h},
$$

and the preconditioned Richardson iterations converge with a rate independent of $h$.

Proof. The proof is similar to that of Theorem 7.2 in [3]. However, for the reader's convenience we will give it in complete detail.

If $\nu$ is an eigenvalue of $\mathrm{S}_{2, h}^{-1}\left(\mathrm{~S}_{1, h}+\mathrm{S}_{2, h}\right)=I+\mathrm{S}_{2, h}^{-1} \mathrm{~S}_{1, h}$, we can write $\nu=1+\kappa$, where $\kappa$ is an eigenvalue of $\mathrm{S}_{2, h}^{-1} \mathrm{~S}_{1, h}$. The corresponding eigenvector $\boldsymbol{\gamma} \in \mathbf{C}^{M_{h}}$, $\boldsymbol{\gamma} \neq \mathbf{0}$, satisfies $\mathrm{S}_{1, h} \gamma=\kappa \mathrm{S}_{2, h} \gamma ;$ therefore,

$$
\left\langle\left\langle\mathcal{S}_{1, h} \gamma_{h}, \gamma_{h}\right\rangle\right\rangle_{h}=\kappa\left\langle\left\langle\mathcal{S}_{2, h} \gamma_{h}, \gamma_{h}\right\rangle\right\rangle_{h}
$$

where $\gamma_{h} \in X_{h}$ is the function defined in (3.23). 
Writing $\kappa=\kappa_{1}+i \kappa_{2}, \kappa_{1}, \kappa_{2} \in \mathbf{R}$, we have

$$
\begin{aligned}
& \operatorname{Re}\left\langle\left\langle\mathcal{S}_{1, h} \boldsymbol{\gamma}_{h}, \boldsymbol{\gamma}_{h}\right\rangle\right\rangle_{h}=\kappa_{1} \operatorname{Re}\left\langle\left\langle\mathcal{S}_{2, h} \gamma_{h}, \boldsymbol{\gamma}_{h}\right\rangle\right\rangle_{h}-\kappa_{2} \operatorname{Im}\left\langle\left\langle\mathcal{S}_{2, h} \boldsymbol{\gamma}_{h}, \boldsymbol{\gamma}_{h}\right\rangle\right\rangle_{h}, \\
& \operatorname{Im}\left\langle\left\langle\mathcal{S}_{1, h} \boldsymbol{\gamma}_{h}, \boldsymbol{\gamma}_{h}\right\rangle\right\rangle_{h}=\kappa_{1} \operatorname{Im}\left\langle\left\langle\mathcal{S}_{2, h} \gamma_{h}, \gamma_{h}\right\rangle\right\rangle_{h}+\kappa_{2} \operatorname{Re}\left\langle\left\langle\mathcal{S}_{2, h} \gamma_{h}, \gamma_{h}\right\rangle\right\rangle_{h} .
\end{aligned}
$$

Multiply now these equations by $\operatorname{Re}\left\langle\left\langle\mathcal{S}_{2, h} \gamma_{h}, \gamma_{h}\right\rangle\right\rangle_{h}$ and $\operatorname{Im}\left\langle\left\langle\mathcal{S}_{2, h} \gamma_{h}, \gamma_{h}\right\rangle\right\rangle_{h}$, respectively: by adding the results we find

$$
\begin{aligned}
\kappa_{1}\left[\left(\operatorname{Re}\left\langle\left\langle\mathcal{S}_{2, h} \gamma_{h}, \gamma_{h}\right\rangle\right\rangle_{h}\right)^{2}+\right. & \left.\left(\operatorname{Im}\left\langle\left\langle\mathcal{S}_{2, h} \gamma_{h}, \gamma_{h}\right\rangle\right\rangle_{h}\right)^{2}\right] \\
= & \operatorname{Re}\left\langle\left\langle\mathcal{S}_{1, h} \gamma_{h}, \gamma_{h}\right\rangle\right\rangle_{h} \operatorname{Re}\left\langle\left\langle\mathcal{S}_{2, h} \gamma_{h}, \gamma_{h}\right\rangle\right\rangle_{h} \\
& +\operatorname{Im}\left\langle\left\langle\mathcal{S}_{1, h} \gamma_{h}, \gamma_{h}\right\rangle\right\rangle_{h} \operatorname{Im}\left\langle\left\langle\mathcal{S}_{2, h} \gamma_{h}, \gamma_{h}\right\rangle\right\rangle_{h} .
\end{aligned}
$$

From (3.25) we have that $\left|\left\langle\left\langle\mathcal{S}_{2, h} \gamma_{h}, \gamma_{h}\right\rangle\right\rangle_{h}\right| \neq 0$; therefore, (3.26) yields $\kappa_{1} \geq 0$.

On the other hand, from (3.24), (3.25) it follows

$$
|\kappa|^{2}=\frac{\left|\left\langle\left\langle\mathcal{S}_{1, h} \gamma_{h}, \gamma_{h}\right\rangle\right\rangle_{h}\right|^{2}}{\left|\left\langle\left\langle\mathcal{S}_{2, h} \gamma_{h}, \gamma_{h}\right\rangle\right\rangle_{h}\right|^{2}} \leq\left(\frac{C_{1}}{C_{2}}\right)^{2}
$$

therefore,

$$
2 \frac{\operatorname{Re} \nu}{|\nu|^{2}}=2 \frac{1+\kappa_{1}}{1+2 \kappa_{1}+|\kappa|^{2}} \geq 2 \frac{1+\kappa_{1}}{1+2 \kappa_{1}+\left(\frac{C_{1}}{C_{2}}\right)^{2}} .
$$

Notice now that the function

$$
F(\xi):=2 \frac{1+\xi}{1+2 \xi+\left(\frac{C_{1}}{C_{2}}\right)^{2}}
$$

is strictly increasing when $C_{1}>C_{2}$, strictly decreasing when $C_{1}<C_{2}$ and constantly equal to 1 when $C_{1}=C_{2}$. Moreover,

$$
F(0)=\frac{2 C_{2}^{2}}{C_{1}^{2}+C_{2}^{2}}, \quad \lim _{\xi \rightarrow \infty} F(\xi)=1 ;
$$

hence, $C^{*}$ is the infimum of $F$ for $\xi \geq 0$.

The proof of the convergence of the iterations (3.19) reduces now to verify that the operators $\mathcal{S}_{1, h}$ and $\mathcal{S}_{2, h}$ satisfy the assumptions of Theorem 3.1, i.e., (3.24)(3.26).

Noting that

$$
\begin{aligned}
\operatorname{Re}\left\langle\left\langle\mathcal{S}_{j, h} \gamma_{h}, \gamma_{h}\right\rangle\right\rangle_{h} & =\int_{\Omega_{j}} \mu^{-1}\left|\operatorname{rot} \mathbf{E}_{j, \Gamma}^{h} \gamma_{h}\right|^{2}, \\
\operatorname{Im}\left\langle\left\langle\mathcal{S}_{j, h} \gamma_{h}, \gamma_{h}\right\rangle\right\rangle_{h} & =\alpha \int_{\Omega_{j}} \sigma\left|\mathbf{E}_{j, \Gamma}^{h} \gamma_{h}\right|^{2},
\end{aligned}
$$

estimate (3.26) is trivially satisfied.

By using the coerciveness of $a_{2}(\cdot, \cdot)$ and the following tangential trace inequality (see Alonso and Valli [2])

$$
\left\|\left(\mathbf{n}_{\Gamma} \times \mathbf{v}\right)_{\mid \Gamma}\right\|_{\mathcal{X}_{\Gamma}}^{2} \leq C^{*}\|\mathbf{v}\|_{H\left(\operatorname{rot} ; \Omega_{2}\right)}^{2}
$$

we have

$$
\left|\left\langle\left\langle\mathcal{S}_{2, h} \gamma_{h}, \gamma_{h}\right\rangle\right\rangle_{h}\right| \geq C\left(\left\|\mathbf{E}_{2, \Gamma}^{h} \gamma_{h}\right\|_{0, \Omega_{2}}^{2}+\left\|\operatorname{rot} \mathbf{E}_{2, \Gamma}^{h} \gamma_{h}\right\|_{0, \Omega_{2}}^{2}\right) \geq \frac{C}{C^{*}}\left\|\gamma_{h}\right\|_{\mathcal{X}_{\Gamma}}^{2} ;
$$

hence, (3.25) holds. 
The proof of (3.24) is more difficult. The crucial point is the proof of the continuity of the extension operator $\mathbf{E}_{1, \Gamma}^{h}$ uniformly with respect to $h$. Let us start by introducing for each $\delta>0$ the space

$$
\mathcal{X}_{\partial \Omega}^{\delta}:=\left\{\boldsymbol{\eta} \in\left(H^{\delta}(\partial \Omega)\right)^{3} \mid(\boldsymbol{\eta} \cdot \mathbf{n})_{\mid \partial \Omega}=0 \text { and } \operatorname{div}_{\tau} \boldsymbol{\eta} \in H^{\delta}(\partial \Omega)\right\},
$$

and for each $r>0$ the space

$$
H^{r}(\operatorname{rot} ; \Omega):=\left\{\mathbf{v} \in\left(H^{r}(\Omega)\right)^{3} \mid \operatorname{rot} \mathbf{v} \in\left(H^{r}(\Omega)\right)^{3}\right\},
$$

endowed with the following norms, respectively:

$$
\begin{aligned}
& \|\boldsymbol{\eta}\|_{\mathcal{X}_{\partial \Omega}^{\delta}}:=\left(\|\boldsymbol{\eta}\|_{\delta, \partial \Omega}^{2}+\left\|\operatorname{div}_{\tau} \boldsymbol{\eta}\right\|_{\delta, \partial \Omega}^{2}\right)^{1 / 2} \\
& \|\mathbf{v}\|_{H^{r}(\operatorname{rot} ; \Omega)}:=\left(\|\mathbf{v}\|_{r, \Omega}^{2}+\|\operatorname{rot} \mathbf{v}\|_{r, \Omega}^{2}\right)^{1 / 2} .
\end{aligned}
$$

Let us denote by $\mathbf{F}_{1, \Gamma}: \mathcal{X}_{\Gamma} \rightarrow V_{1}$ the extension operator which at each $\gamma \in \mathcal{X}_{\Gamma}$ associates $\mathbf{F}_{1, \Gamma} \boldsymbol{\gamma}$ such that

$$
\left\{\begin{array}{l}
\mathbf{F}_{1, \Gamma} \boldsymbol{\gamma} \in V_{1}: \\
\left(\left(\mathbf{F}_{1, \Gamma} \boldsymbol{\gamma}, \mathbf{v}_{1}\right)\right)_{\Omega_{1}}=0 \quad \forall \mathbf{v}_{1} \in H_{0}\left(\operatorname{rot} ; \Omega_{1}\right) \\
\left(\mathbf{n}_{\Gamma} \times \mathbf{F}_{1, \Gamma} \boldsymbol{\gamma}\right)_{\mid \Gamma}=\gamma
\end{array}\right.
$$

where

$$
\left(\left(\mathbf{w}_{1}, \mathbf{v}_{1}\right)\right)_{\Omega_{1}}:=\int_{\Omega_{1}}\left(\operatorname{rot} \mathbf{w}_{1} \cdot \operatorname{rot} \overline{\mathbf{v}_{1}}+\mathbf{w}_{1} \cdot \overline{\mathbf{v}_{1}}\right) .
$$

The existence of such an operator is guaranteed provided that we can characterize $\mathcal{X}_{\Gamma}$ as the space of tangential traces on $\Gamma$ of $V_{1}$. In that case, as a consequence of the closed graph theorem, it easily follows that $\mathbf{F}_{1, \Gamma}$ is a continuous operator, i.e.,

$$
\left\|\mathbf{F}_{1, \Gamma} \gamma\right\|_{H\left(\operatorname{rot} ; \Omega_{1}\right)} \leq C_{0}\|\gamma\|_{\mathcal{X}_{\Gamma}} \quad \forall \gamma \in \mathcal{X}_{\Gamma} .
$$

The needed characterization result on $\mathcal{X}_{\Gamma}$ was proved in [2], under the assumption that $\Gamma$ is either a $C^{1,1}$ surface or a convex polyhedral portion of $\partial \Omega_{1}$.

Finally, introduce the extension operator $\mathbf{F}_{1, \Gamma}^{h}: \mathcal{X}_{h, \Gamma} \rightarrow V_{1, h}$, which is the finite dimensional counterpart of $\mathbf{F}_{1, \Gamma}$ :

$$
\left\{\begin{array}{l}
\mathbf{F}_{1, \Gamma}^{h} \gamma_{h} \in V_{1, h}: \\
\left(\left(\mathbf{F}_{1, \Gamma}^{h} \gamma_{h}, \mathbf{v}_{1, h}\right)\right)_{\Omega_{1}}=0 \quad \forall \mathbf{v}_{1, h} \in V_{1, h}^{0} \\
\left(\mathbf{n}_{\Gamma} \times \mathbf{F}_{1, \Gamma}^{h} \gamma_{h}\right)_{\mid \Gamma}=\gamma_{h} .
\end{array}\right.
$$

We need the following regularity result, which is Proposition 3.7 in Amrouche, Bernardi, Dauge and Girault [5]. Let us set

$$
\begin{aligned}
& X_{T}:=H(\operatorname{rot} ; \Omega) \cap H_{0}(\operatorname{div} ; \Omega), \\
& X_{N}:=H_{0}(\operatorname{rot} ; \Omega) \cap H(\operatorname{div} ; \Omega),
\end{aligned}
$$

both endowed with the norm

$$
\|\mathbf{v}\|_{0, \Omega}+\|\operatorname{div} \mathbf{v}\|_{0, \Omega}+\|\operatorname{rot} \mathbf{v}\|_{0, \Omega} .
$$

Theorem 3.2. Let $\Omega$ be a Lipschitz polyhedron. Then there exists $s_{\Omega} \in(1 / 2,1)$ such that the spaces $X_{T}$ and $X_{N}$ are both continuously imbedded in $\left(H^{s_{\Omega}}(\Omega)\right)^{3}$. 
We notice that $s_{\Omega}$ only depends on the geometry of $\Omega$. It is related to the exponent of maximal regularity of the solutions to the Laplace operator with $L^{2}(\Omega)$ on the right-hand side and homogeneous Dirichlet or Neumann boundary datum (see Amrouche, Bernardi, Dauge and Girault [5], Remark 3.8).

The proof of (3.24) is based on the following three theorems, which will be proven in the Sections 4,5 and 6. From now on the subdomain $\Omega_{1}$ is always assumed to be a Lipschitz polyhedron. Finally, set $\kappa_{\Omega}:=s_{\Omega}-1 / 2$, where $s_{\Omega}$ is as in Theorem 3.2 .

Theorem A. Assume that $\Gamma$ is a convex portion of $\partial \Omega_{1}$. Given $\delta \in\left(0, \kappa_{\Omega_{1}}\right]$, there exists $K_{1}>0$ such that for all $\gamma \in \mathcal{X}_{\Gamma}$ with $\widetilde{\gamma} \in \mathcal{X}_{\partial \Omega_{1}}^{\delta}$ one has $\mathbf{F}_{1, \Gamma} \gamma \in$ $H^{1 / 2+\delta}\left(\operatorname{rot} ; \Omega_{1}\right)$ and

$$
\left\|\mathbf{F}_{1, \Gamma} \gamma\right\|_{H^{\frac{1}{2}+\delta}\left(\operatorname{rot} ; \Omega_{1}\right)} \leq K_{1}\|\widetilde{\gamma}\|_{\mathcal{X}_{\partial \Omega_{1}}^{\delta}}
$$

Here, as usual, $\widetilde{\gamma}$ denotes the extension of $\boldsymbol{\gamma}$ by $\mathbf{0}$ on $\partial \Omega_{1} \backslash \Gamma$.

Theorem B. Let $\mathcal{T}_{h}$ be a regular family of triangulations. Assume that $\gamma_{h} \in \mathcal{X}_{\Gamma, h}$ and that $\mathbf{F}_{1, \Gamma} \gamma_{h} \in H^{r}\left(\operatorname{rot} ; \Omega_{1}\right)$ for a certain $r \in(1 / 2,1)$. Then there exists a constant $K_{2}>0$, independent of $h$, such that

$$
\left\|\mathbf{F}_{1, \Gamma} \gamma_{h}-\mathbf{F}_{1, \Gamma}^{h} \gamma_{h}\right\|_{H\left(\operatorname{rot} ; \Omega_{1}\right)} \leq K_{2} h^{r}\left\|\mathbf{F}_{1, \Gamma} \gamma_{h}\right\|_{H^{r}\left(\operatorname{rot} ; \Omega_{1}\right)} \quad \forall \gamma_{h} \in \mathcal{X}_{\Gamma, h} .
$$

Theorem C. Let $\mathcal{M}_{h}$ be the family of triangulations of $\partial \Omega_{1}$ induced by $\mathcal{T}_{h}$. Assume that $\mathcal{M}_{h}$ is quasi-uniform. Then for each $\epsilon \in(0,1 / 2)$ there exists a constant $K_{3}>0$, independent of $h$, such that

$$
\left\|\widetilde{\gamma}_{h}\right\|_{\mathcal{X}_{\partial \Omega_{1}}^{\varepsilon}} \leq K_{3} h^{-\frac{1}{2}-\varepsilon}\left\|\gamma_{h}\right\|_{\mathcal{X}_{\Gamma}} \quad \forall \gamma_{h} \in \mathcal{X}_{\Gamma, h}
$$

Once we have established these results, we are in a condition to prove the following

Proposition 3.3. Assume that $\Gamma$ is convex portion of $\partial \Omega_{1}$ and that $\mathcal{M}_{h}$ is a quasiuniform family of triangulations of $\partial \Omega_{1}$. Then there exists a constant $K_{4}>0$, independent of $h$, such that

$$
\left|\left\langle\left\langle\mathcal{S}_{1, h} \boldsymbol{\gamma}_{h}, \boldsymbol{\eta}_{h}\right\rangle\right\rangle_{h}\right| \leq K_{4}|| \gamma_{h} \mid \mathcal{X}_{\Gamma}\left\|\boldsymbol{\eta}_{h}\right\|_{\mathcal{X}_{\Gamma}} \quad \forall \gamma_{h}, \boldsymbol{\eta}_{h} \in \mathcal{X}_{\Gamma, h}
$$

which is estimate (3.24).

Proof. From the definition of the Steklov-Poincaré operator $\mathcal{S}_{1, h}$ we have

$$
\begin{aligned}
\left|\left\langle\left\langle\mathcal{S}_{1, h} \gamma_{h}, \boldsymbol{\eta}_{h}\right\rangle\right\rangle_{h}\right| & =\left|a_{1}\left(\mathbf{E}_{1, \Gamma}^{h} \boldsymbol{\gamma}_{h}, \mathbf{E}_{1, \Gamma}^{h} \boldsymbol{\eta}_{h}\right)\right| \\
& \leq \beta_{1}|| \mathbf{E}_{1, \Gamma}^{h} \gamma_{h}\left\|_{H\left(\operatorname{rot} ; \Omega_{1}\right)}|| \mathbf{E}_{1, \Gamma}^{h} \boldsymbol{\eta}_{h}\right\|_{H\left(\operatorname{rot} ; \Omega_{1}\right)}
\end{aligned}
$$

where $\beta_{1}>0$ is the continuity constant of $a_{1}(\cdot, \cdot)$. Therefore, the proof is complete if we show that there exists a constant $C>0$, independent of $h$, such that

$$
\left\|\mathbf{E}_{1, \Gamma}^{h} \gamma_{h}\right\|_{H\left(\operatorname{rot} ; \Omega_{1}\right)} \leq C\left\|\gamma_{h}\right\|_{\mathcal{X}_{\Gamma}} \quad \forall \gamma_{h} \in \mathcal{X}_{\Gamma, h}
$$

Taking in (3.13) the test function $\mathbf{v}_{1, h}=\mathbf{E}_{1, \Gamma}^{h} \gamma_{h}-\mathbf{F}_{1, \Gamma}^{h} \gamma_{h}$ we have

$$
a_{1}\left(\mathbf{E}_{1, \Gamma}^{h} \boldsymbol{\gamma}_{h}, \mathbf{E}_{1, \Gamma}^{h} \boldsymbol{\gamma}_{h}\right)=a_{1}\left(\mathbf{E}_{1, \Gamma}^{h} \boldsymbol{\gamma}_{h}, \mathbf{F}_{1, \Gamma}^{h} \boldsymbol{\gamma}_{h}\right)
$$

Hence

$$
\left\|\mathbf{E}_{1, \Gamma}^{h} \gamma_{h}\right\|_{H\left(\operatorname{rot} ; \Omega_{1}\right)} \leq \frac{\beta_{1}}{\alpha_{1}}\left\|\mathbf{F}_{1, \Gamma}^{h} \gamma_{h}\right\|_{H\left(\operatorname{rot} ; \Omega_{1}\right)},
$$

where $\alpha_{1}$ is the coerciveness constant of the bilinear form $a_{1}(\cdot, \cdot)$. 
Moreover

$$
\left\|\mathbf{F}_{1, \Gamma}^{h} \gamma_{h}\right\|_{H\left(\operatorname{rot} ; \Omega_{1}\right)} \leq\left\|\mathbf{F}_{1, \Gamma}^{h} \gamma_{h}-\mathbf{F}_{1, \Gamma} \gamma_{h}\right\|_{H\left(\operatorname{rot} ; \Omega_{1}\right)}+\left\|\mathbf{F}_{1, \Gamma} \gamma_{h}\right\|_{H\left(\operatorname{rot} ; \Omega_{1}\right)}
$$

therefore, from (3.27) we only have to estimate the first term in (3.29).

At first, remark that $\gamma_{h} \in \mathcal{X}_{\Gamma, h}$ yields $\widetilde{\gamma_{h}} \in \mathcal{X}_{\partial \Omega_{1}}^{\delta}$ for each $\delta \in(0,1 / 2)$, as both $\widetilde{\gamma_{h}}$ and $\operatorname{div}_{\tau} \widetilde{\gamma_{h}}$ are (discontinuous) piecewise-polynomial. Therefore, from Theorem A we have that $\mathbf{F}_{1, \Gamma} \gamma_{h} \in H^{1 / 2+\delta}\left(\operatorname{rot} ; \Omega_{1}\right)$ for any $\delta \in\left(0, \kappa_{\Omega_{1}}\right]$. Using also Theorem B (where $r=1 / 2+\delta$ ) we have

$$
\begin{aligned}
\left\|\mathbf{F}_{1, \Gamma}^{h} \boldsymbol{\gamma}_{h}-\mathbf{F}_{1, \Gamma} \boldsymbol{\gamma}_{h}\right\|_{H\left(\mathrm{rot} ; \Omega_{1}\right)} & \leq K_{2} h^{\frac{1}{2}+\delta}\left\|\mathbf{F}_{1, \Gamma} \boldsymbol{\gamma}_{h}\right\|_{H^{\frac{1}{2}+\delta}\left(\operatorname{rot} ; \Omega_{1}\right)} \\
& \leq K_{1} K_{2} h^{\frac{1}{2}+\delta}\left\|\widetilde{\gamma}_{h}\right\|_{\mathcal{X}_{\partial \Omega_{1}}^{\delta}} .
\end{aligned}
$$

Now we can apply Theorem $\mathrm{C}($ for $\epsilon=\delta$ ) and we find

$$
\left\|\mathbf{F}_{1, \Gamma}^{h} \gamma_{h}-\mathbf{F}_{1, \Gamma} \gamma_{h}\right\|_{H\left(\operatorname{rot} ; \Omega_{1}\right)} \leq K_{1} K_{2} K_{3}\left\|\gamma_{h}\right\|_{\mathcal{X}_{\Gamma}}
$$

The proof follows from (3.27)-(3.30).

Remark 3.4. In Theorem $\mathrm{A}$ and in Proposition 3.3 the assumption on $\Gamma$ is only needed to assure that $\mathcal{X}_{\Gamma}$ is the space of tangential traces on $\Gamma$ of $V_{1}$.

\section{Proof of Theorem A}

Let us introduce the finite dimensional spaces

$$
\begin{aligned}
\mathcal{H}(e) & :=\left\{\boldsymbol{\omega} \in\left(L^{2}(\Omega)\right)^{3} \mid \operatorname{rot} \boldsymbol{\omega}=\mathbf{0}, \operatorname{div} \boldsymbol{\omega}=0,(\mathbf{n} \times \boldsymbol{\omega})_{\mid \partial \Omega}=\mathbf{0}\right\}, \\
\mathcal{H}(m) & :=\left\{\varrho \in\left(L^{2}(\Omega)\right)^{3} \mid \operatorname{rot} \varrho=\mathbf{0}, \operatorname{div} \varrho=0,(\varrho \cdot \mathbf{n})_{\mid \partial \Omega}=0\right\} .
\end{aligned}
$$

We start recalling the following theorems, whose proof can be essentially found in Saranen [16] (see also Valli [17]).

Theorem 4.1. Let $\Omega \subset \mathbf{R}^{3}$ be a bounded domain with Lipschitz boundary $\partial \Omega$. Each function $\mathbf{w} \in\left(L^{2}(\Omega)\right)^{3}$ can be written as

$$
\mathbf{w}=\operatorname{rot} \mathbf{p}+\nabla q+\sum_{k=1}^{n} \alpha_{k} \boldsymbol{\varrho}_{k},
$$

where $\mathbf{p}$ satisfies

$$
\begin{cases}\operatorname{rot} \operatorname{rot} \mathbf{p}=\operatorname{rot} \mathbf{w} & \text { in } \Omega \\ \operatorname{div} \mathbf{p}=0 & \text { in } \Omega \\ (\mathbf{n} \times \mathbf{p})_{\mid \partial \Omega}=\mathbf{0} & \text { on } \partial \Omega \\ (\mathbf{p}, \boldsymbol{\omega})=0 & \forall \boldsymbol{\omega} \in \mathcal{H}(e),\end{cases}
$$

$q$ satisfies

$$
\begin{cases}\Delta q=\operatorname{div} \mathbf{w} & \text { in } \Omega \\ \left(\frac{\partial q}{\partial n}\right)_{\mid \partial \Omega}=(\mathbf{w} \cdot \mathbf{n})_{\mid \partial \Omega} & \text { on } \partial \Omega \\ \int_{\Omega} q=0, & \end{cases}
$$


the functions $\left\{\varrho_{k}\right\}_{k=1}^{n}$ are an orthonormal basis of $\mathcal{H}(m)$, and the coefficients $\alpha_{k}$ are given by $\alpha_{k}=\left(\mathbf{w}, \varrho_{k}\right), k=1, \ldots, n$.

Theorem 4.2. Let $\Omega \subset \mathbf{R}^{3}$ be a bounded domain with Lipschitz boundary $\partial \Omega$. Then there exists a constant $C_{\Omega}>0$ such that

$$
\|\mathbf{w}\|_{0, \Omega} \leq C_{\Omega}\left(\|\operatorname{rot} \mathbf{w}\|_{0, \Omega}+\|\operatorname{div} \mathbf{w}\|_{0, \Omega}\right) \quad \forall \mathbf{w} \in X_{T} \cap \mathcal{H}(m)^{\perp} .
$$

We are now in a position to prove some auxiliary results, which are interesting on their own as they are regularity results for harmonic fields. In the particular case in which the parameter $\delta$ is equal to 0 , Costabel [6] proved the same regularity result for a simply connected Lipschitz domain with connected boundary.

Theorem 4.3 (Regularity for Dirichlet harmonic fields). Let $\Omega$ be a Lipschitz polyhedron. Then for each $\delta \in(0,1 / 2)$ the space

$$
W:=\left\{\mathbf{w} \in H(\operatorname{rot} ; \Omega) \cap H(\operatorname{div} ; \Omega) \mid(\mathbf{n} \times \mathbf{w})_{\mid \partial \Omega} \in\left(H^{\delta}(\partial \Omega)\right)^{3}\right\}
$$

is continuously imbedded in $\left(H^{1 / 2+\epsilon_{*}}(\Omega)\right)^{3}$, where $\epsilon_{*}:=\min \left(\delta, \kappa_{\Omega}\right)$.

Proof. From Theorem 4.1 each function $\mathbf{w} \in W$ can be written as

$$
\mathbf{w}=\operatorname{rot} \mathbf{p}+\nabla q+\sum_{k=1}^{n} \alpha_{k} \varrho_{k} .
$$

Since $\operatorname{rot} \operatorname{rot} \mathbf{p}=\operatorname{rot} \mathbf{w} \in\left(L^{2}(\Omega)\right)^{3}$, and $(\mathbf{n} \times \mathbf{p})_{\mid \partial \Omega}=\mathbf{0}$ yields $(\operatorname{rot} \mathbf{p} \cdot \mathbf{n})_{\mid \partial \Omega}=$ $-\operatorname{div}_{\tau}(\mathbf{n} \times \mathbf{p})_{\mid \partial \Omega}=0$, we have that $\operatorname{rot} \mathbf{p} \in X_{T}$. Moreover, $\varrho_{k} \in \mathcal{H}(m) \subset X_{T}$; hence, from Theorem 3.2 we find that $\operatorname{rot} \mathbf{p}$ and each $\varrho_{k}$ belong to $\left(H^{1 / 2+\kappa_{\Omega}}(\Omega)\right)^{3}$. From $\alpha_{k}=\left(\mathbf{w}, \varrho_{k}\right)$ it follows at once that

$$
\sum_{k=1}^{n}\left|\alpha_{k}\right|\left\|\boldsymbol{\varrho}_{k}\right\|_{\frac{1}{2}+\kappa_{\Omega}, \Omega} \leq C\|\mathbf{w}\|_{0, \Omega}
$$

Moreover, it is easily verified that $\operatorname{rot} \mathbf{p} \in \mathcal{H}(m)^{\perp}$; hence, from Theorems 3.2 and 4.2 we have

$$
\begin{aligned}
\|\operatorname{rot} \mathbf{p}\|_{\frac{1}{2}+\kappa_{\Omega}, \Omega} & \leq C\|\operatorname{rot} \mathbf{p}\|_{X_{T}} \\
& \leq C\left(1+C_{\Omega}\right)\left(\|\operatorname{rot} \operatorname{rot} \mathbf{p}\|_{0, \Omega}+\|\operatorname{div} \operatorname{rot} \mathbf{p}\|_{0, \Omega}\right) \\
& =C\left(1+C_{\Omega}\right)\|\operatorname{rot} \mathbf{w}\|_{0, \Omega} .
\end{aligned}
$$

On the other hand, from

$$
\nabla q=(\nabla q \cdot \mathbf{n})_{\mid \partial \Omega} \mathbf{n}-\mathbf{n} \times(\mathbf{n} \times \nabla q)_{\mid \partial \Omega}
$$

we have

$$
\nabla_{\tau} q_{\mid \partial \Omega}=-\mathbf{n} \times(\mathbf{n} \times \nabla q)_{\mid \partial \Omega},
$$

and therefore

$$
\nabla_{\tau} q_{\mid \partial \Omega}=-\mathbf{n} \times\left((\mathbf{n} \times \mathbf{w})_{\mid \partial \Omega}-(\mathbf{n} \times \operatorname{rot} \mathbf{p})_{\mid \partial \Omega}-\left(\mathbf{n} \times \sum_{k=1}^{n} \alpha_{k} \boldsymbol{\varrho}_{k}\right)_{\mid \partial \Omega}\right) .
$$

The unit normal vector $\mathbf{n}$ is piecewise constant, as $\Omega$ is a polyhedron; hence,

$$
\nabla_{\tau} q_{\mid \partial \Omega} \in\left(H^{\epsilon_{*}}(\Omega)\right)^{3} .
$$

From $q \in H^{1}(\Omega)$ we also have that $q_{\mid \partial \Omega} \in H^{1 / 2}(\partial \Omega) \subset L^{2}(\partial \Omega)$; therefore, we conclude that

$$
q_{\mid \partial \Omega} \in H^{1+\epsilon_{*}}(\partial \Omega)
$$


and we find the estimate

$$
\begin{aligned}
\left\|q_{\mid \partial \Omega}\right\|_{1+\varepsilon_{*}, \partial \Omega} \leq C\left(\|q\|_{0, \partial \Omega}\right. & \left.+\left\|\nabla_{\tau} q_{\mid \partial \Omega}\right\|_{\varepsilon_{*}, \partial \Omega}\right) \leq C\left(\|q\|_{1, \Omega}+\left\|\nabla_{\tau} q_{\mid \partial \Omega}\right\|_{\varepsilon_{*}, \partial \Omega}\right) \\
\leq C\left(\|\mathbf{w}\|_{0, \Omega}\right. & +\left\|(\mathbf{n} \times \mathbf{w})_{\mid \partial \Omega}\right\|_{\delta, \partial \Omega} \\
& \left.+\|\operatorname{rot} \mathbf{p}\|_{\frac{1}{2}+\kappa_{\Omega}, \Omega}+\sum_{k=1}^{n}\left|\alpha_{k}\right|\left\|\boldsymbol{\varrho}_{k}\right\|_{\frac{1}{2}+\kappa_{\Omega}, \Omega}\right) .
\end{aligned}
$$

Since $\Delta q=\operatorname{div} \mathbf{w} \in L^{2}(\Omega)$, from the regularity results for the Dirichlet boundary value problem for the Laplace operator it follows that $q \in H^{3 / 2+\epsilon_{*}}(\Omega)$ (see Dauge [7], Corollary 18.13), and

$$
\|\nabla q\|_{\frac{1}{2}+\varepsilon_{*}, \Omega} \leq\|q\|_{\frac{3}{2}+\varepsilon_{*}, \Omega} \leq C\left(\|\operatorname{div} \mathbf{w}\|_{0, \Omega}+\left\|q_{\mid \partial \Omega}\right\|_{1+\varepsilon_{*}, \partial \Omega}\right) .
$$

From the representation formula (4.1) we finally have $\mathbf{w} \in\left(H^{3 / 2+\epsilon_{*}}(\Omega)\right)^{3}$ and from $(4.5)-(4.7)$

$$
\begin{aligned}
\|\mathbf{w}\|_{\frac{1}{2}+\varepsilon_{*}, \Omega} & \leq\|\operatorname{rot} \mathbf{p}\|_{\frac{1}{2}+\kappa_{\Omega}, \Omega}+\|\nabla q\|_{\frac{1}{2}+\varepsilon_{*}, \Omega}+\sum_{k=1}^{n}\left|\alpha_{k}\right|\left\|\varrho_{k}\right\|_{\frac{1}{2}+\kappa_{\Omega}, \Omega} \\
& \leq C\left(\|\mathbf{w}\|_{0, \Omega}+\|\operatorname{rot} \mathbf{w}\|_{0, \Omega}+\|\operatorname{div} \mathbf{w}\|_{0, \Omega}+\left\|(\mathbf{n} \times \mathbf{w})_{\mid \partial \Omega}\right\|_{\delta, \partial \Omega}\right)
\end{aligned}
$$

which concludes the proof.

A similar result is the following

Theorem 4.4 (Regularity for Neumann harmonic fields). Let $\Omega$ be a Lipschitz polyhedron. Then for each $\delta \in(0,1 / 2)$ the space

$$
V:=\left\{\mathbf{w} \in H(\operatorname{rot} ; \Omega) \cap H(\operatorname{div} ; \Omega) \mid(\mathbf{n} \cdot \mathbf{w})_{\mid \partial \Omega} \in H^{\delta}(\partial \Omega)\right\}
$$

is continuously imbedded in $\left(H^{1 / 2+\epsilon_{*}}(\Omega)\right)^{3}$, where $\epsilon_{*}:=\min \left(\delta, \kappa_{\Omega}\right)$.

Proof. As in the proof of Theorem 4.3, we use the representation formula (4.1). The first and the third term can be treated as done there; hence, we have only to check the regularity of $q$.

It is the solution of a Neumann boundary value problem for the Laplace operator with $L^{2}(\Omega)$ right-hand side, and $H^{\delta}(\partial \Omega)$ Neumann datum. As a consequence of Corollary 23.5 in Dauge [7] we have that $q \in H^{3 / 2+\epsilon_{*}}(\Omega)$ and

$$
\|\nabla q\|_{\frac{1}{2}+\varepsilon_{*}, \Omega} \leq C\left(\|\operatorname{div} \mathbf{w}\|_{0, \Omega}+\left\|(\mathbf{n} \cdot \mathbf{w})_{\mid \partial \Omega}\right\|_{\delta, \partial \Omega}\right) .
$$

Using (4.5), (4.6) we finally have

$$
\begin{aligned}
\|\mathbf{w}\|_{\frac{1}{2}+\varepsilon_{*}, \Omega} & \leq\|\operatorname{rot} \mathbf{p}\|_{\frac{1}{2}+\kappa_{\Omega}, \Omega}+\|\nabla q\|_{\frac{1}{2}+\varepsilon_{*}, \Omega}+\sum_{k=1}^{n}\left|\alpha_{k}\right|\left\|\boldsymbol{\varrho}_{k}\right\|_{\frac{1}{2}+\kappa_{\Omega}, \Omega} \\
& \leq C\left(\|\mathbf{w}\|_{0, \Omega}+\|\operatorname{rot} \mathbf{w}\|_{0, \Omega}+\|\operatorname{div} \mathbf{w}\|_{0, \Omega}+\left\|(\mathbf{n} \cdot \mathbf{w})_{\mid \partial \Omega}\right\|_{\delta, \partial \Omega}\right)
\end{aligned}
$$

and the proof is concluded.

We are now in a position to give the proof of Theorem A.

Proof of Theorem A. $\mathbf{F}_{1, \Gamma} \boldsymbol{\gamma} \in H\left(\operatorname{rot} ; \Omega_{1}\right)$ satisfies

$$
\operatorname{rot} \operatorname{rot} \mathbf{F}_{1, \Gamma} \boldsymbol{\gamma}+\mathbf{F}_{1, \Gamma} \boldsymbol{\gamma}=\mathbf{0} \text { in } \Omega_{1}
$$

therefore, $\operatorname{div} \mathbf{F}_{1, \Gamma} \boldsymbol{\gamma}=0$ in $\Omega_{1}$. We can apply Theorem 4.3 and we find that $\mathbf{F}_{1, \Gamma} \boldsymbol{\gamma} \in H^{1 / 2+\delta}\left(\Omega_{1}\right)$ and

$$
\left\|\mathbf{F}_{1, \Gamma} \gamma\right\|_{\frac{1}{2}+\delta, \Omega_{1}} \leq C\left(\left\|\mathbf{F}_{1, \Gamma} \gamma\right\|_{H\left(\operatorname{rot} ; \Omega_{1}\right)}+\|\widetilde{\gamma}\|_{\delta, \partial \Omega_{1}}\right) .
$$


Assuming that $\Gamma$ is convex portion of $\partial \Omega_{1}$, from (3.27) we finally have

$$
\left\|\mathbf{F}_{1, \Gamma} \gamma\right\|_{\frac{1}{2}+\delta, \Omega_{1}} \leq C\left(\|\widetilde{\gamma}\|_{\delta, \partial \Omega_{1}}+\left\|\operatorname{div}_{\tau} \widetilde{\gamma}\right\|_{-1 / 2, \partial \Omega_{1}}\right)
$$

Let us denote by $\mathbf{G}_{1, \Gamma} \boldsymbol{\gamma}:=\operatorname{rot} \mathbf{F}_{1, \Gamma} \boldsymbol{\gamma}$. We first notice that

$$
\left(\mathbf{G}_{1, \Gamma} \boldsymbol{\gamma} \cdot \mathbf{n}\right)_{\mid \partial \Omega_{1}}=-\operatorname{div}_{\tau} \widetilde{\gamma} \in H^{\delta}\left(\partial \Omega_{1}\right)
$$

and that $\operatorname{rot} \mathbf{G}_{1, \Gamma} \boldsymbol{\gamma}=-\mathbf{F}_{1, \Gamma} \boldsymbol{\gamma} \in\left(L^{2}\left(\Omega_{1}\right)\right)^{3}$. We can apply Theorem 4.4 and we find that $\mathbf{G}_{1, \Gamma} \boldsymbol{\gamma} \in H^{1 / 2+\delta}\left(\Omega_{1}\right)$ and

$$
\left\|\mathbf{G}_{1, \Gamma} \gamma\right\|_{\frac{1}{2}+\delta, \Omega_{1}} \leq C\left(\left\|\mathbf{G}_{1, \Gamma} \gamma\right\|_{H\left(\operatorname{rot} ; \Omega_{1}\right)}+\left\|\operatorname{div}_{\tau} \widetilde{\gamma}\right\|_{\delta, \partial \Omega_{1}}\right) .
$$

On the other hand, it is at once verified that

$$
\left\|\mathbf{G}_{1, \Gamma} \gamma\right\|_{H\left(\operatorname{rot} ; \Omega_{1}\right)} \leq C|| \mathbf{F}_{1, \Gamma} \gamma \|_{H\left(\operatorname{rot} ; \Omega_{1}\right)} ;
$$

hence, from (3.27)

$$
\left\|\operatorname{rot} \mathbf{F}_{1, \Gamma} \gamma\right\|_{\frac{1}{2}+\delta, \Omega_{1}} \leq C\left(\|\gamma\|_{-1 / 2, \Gamma}+\left\|\operatorname{div}_{\tau} \widetilde{\gamma}\right\|_{\delta, \partial \Omega_{1}}\right) .
$$

From (4.10), (4.11) we have

$$
\left\|\mathbf{F}_{1, \Gamma} \gamma\right\|_{H^{\frac{1}{2}+\delta}\left(\operatorname{rot} ; \Omega_{1}\right)} \leq K_{1}\|\widetilde{\gamma}\|_{\mathcal{X}_{\partial \Omega_{1}}^{\delta}},
$$

which concludes the proof.

\section{Proof of Theorem B}

The proof of Theorem B is based on the estimate for the interpolation error. Let us recall that the finite elements we are going to employ are the Nédélec finite element of first type (however, as we already noticed, the same results hold also for the Nédélec finite element of second type introduced in [13]). They are defined for $k \geq 1$ as

$$
N_{h}^{k}:=\left\{\mathbf{v}_{h} \in H(\operatorname{rot} ; \Omega) \mid \mathbf{v}_{h \mid K} \in R_{k} \forall K \in \mathcal{T}_{h}\right\},
$$

where

$$
R_{k}:=\left(\mathbf{P}_{k-1}\right)^{3} \oplus S_{k}, \quad S_{k}:=\left\{\mathbf{p} \in\left(\mathbf{P}_{k}^{*}\right)^{3} \mid \mathbf{p}(\mathbf{x}) \cdot \mathbf{x}=0\right\}
$$

and $\mathbf{P}_{k}^{*}$ is the space of homogeneous polynomials of degree $k$. The degrees of freedom of $N_{h}^{k}$ are given by

$$
m_{1}(\mathbf{v}):=\left\{\int_{a} \mathbf{v} \cdot \mathbf{t}_{a} q \text { for all } q \in \mathbf{P}_{k-1}(a) \text { for the six edges } a \text { of } K\right\},
$$

where $\mathbf{t}_{a}$ is a unit vector having the same direction as the edge $a$; when $k \geq 2$ one has to add

$$
m_{2}(\mathbf{v}):=\left\{\int_{f}(\mathbf{v} \times \mathbf{n}) \cdot \mathbf{q} \text { for all } \mathbf{q} \in\left(\mathbf{P}_{k-2}(f)\right)^{2} \text { for the four faces } f \text { of } K\right\} ;
$$

and finally for $k \geq 3$ one has to take also

$$
m_{3}(\mathbf{v}):=\left\{\int_{K} \mathbf{v} \cdot \mathbf{q} \text { for all } \mathbf{q} \in\left(\mathbf{P}_{k-3}(K)\right)^{3}\right\} .
$$

Nédélec [12] has proven that these degrees of freedom are "curl-conforming" and determine a unique element of $R_{k}$. Let us denote by $\Pi_{h}^{k}$ the interpolation operator valued in $N_{h}^{k}$. 
Lemma 5.1. The interpolation operator $\Pi_{h}^{k}$ is defined in $H^{r}(\operatorname{rot} ; \Omega)$ for any $r>$ $1 / 2$.

Proof. It is only necessary to see that the moments introduced in (5.2)-(5.4) are well defined.

If $\mathbf{v} \in H^{r}(\operatorname{rot} ; \Omega)$ for $r>1 / 2$, then in particular $\mathbf{v}_{\mid K} \in\left(H^{r}(K)\right)^{3}$ and from the trace theorem $\mathbf{v}_{\mid f} \in\left(L^{2}(f)\right)^{3}$; therefore, the moments $m_{3}(\mathbf{v})$ and $m_{2}(\mathbf{v})$ are defined.

Concerning the moments $m_{1}(\mathbf{v})$, let $a$ be one of the edges of a face $f$. Denote as usual by $\mathbf{n}$ the unit outward normal vector on $\partial K$ and by $\boldsymbol{\nu}$ the unit vector contained in the plane identified by $f$, pointing outward $f$ and normal to $a$. The unit vector $\mathbf{t}_{a}$ can be written as $\mathbf{t}_{a}=\mathbf{n} \times \boldsymbol{\nu}$. Therefore we have

$$
\int_{a} \mathbf{v} \cdot \mathbf{t}_{a} q=\int_{a} \mathbf{v} \cdot(\mathbf{n} \times \boldsymbol{\nu}) q=\int_{a}(\mathbf{v} \times \mathbf{n}) \cdot \boldsymbol{\nu} q .
$$

From the assumption on $\mathbf{v}$ we know $\mathbf{v}_{\mid f} \times \mathbf{n} \in\left(H^{r-1 / 2}(f)\right)^{3}$ and $\operatorname{div}_{\tau}\left(\mathbf{v}_{\mid f} \times \mathbf{n}\right)=$ $(\operatorname{rot} \mathbf{v})_{\mid f} \cdot \mathbf{n} \in H^{r-1 / 2}(f)$; hence, in particular $\mathbf{v}_{\mid f} \times \mathbf{n} \in\left(L^{p}(f)\right)^{3}$ for a suitable $p>2$ and $\operatorname{div}_{\tau}\left(\mathbf{v}_{\mid f} \times \mathbf{n}\right) \in L^{2}(f)$. This easily yields $\left(\left(\mathbf{v}_{\mid f} \times \mathbf{n}\right) \cdot \boldsymbol{\nu}\right)_{\mid \partial f} \in W^{-1 / p, p}(\partial f)$ and then the moments $m_{1}(\mathbf{v})$ are defined by means of a duality argument.

Now we want to prove that

$$
\left\|\mathbf{v}-\Pi_{h}^{k} \mathbf{v}\right\|_{H(\operatorname{rot} ; \Omega)} \leq C h^{\min (r, k)}\|\mathbf{v}\|_{H^{r}(\operatorname{rot} ; \Omega)} \quad \forall \mathbf{v} \in H^{r}(\operatorname{rot} ; \Omega),
$$

where, as before, $r>1 / 2$ and $k \geq 1$.

This result is already known when $r \geq k$, as for any $\mathbf{v} \in H^{k}(\operatorname{rot} ; \Omega)$ Nédélec [12] has proven

$$
\left\|\mathbf{v}-\Pi_{h}^{k} \mathbf{v}\right\|_{0, \Omega} \leq C h^{k}\left(|\mathbf{v}|_{k, \Omega}+|\operatorname{rot} \mathbf{v}|_{k, \Omega}\right),
$$

and Monk [11] has obtained

$$
\left\|\operatorname{rot}\left(\mathbf{v}-\Pi_{h}^{k} \mathbf{v}\right)\right\|_{0, \Omega} \leq C h^{k}\|\operatorname{rot} \mathbf{v}\|_{k, \Omega} .
$$

By following their proofs, it is an easy matter to verify that (5.6) holds also for a positive integer $r, r \leq k-1$. Therefore, we are left with the proof of (5.6) in the case of a non-integer $r, 1 / 2<r<k$.

The reference tetrahedron $\hat{K}$ is the one with vertices $P_{0}:=(0,0,0), P_{1}:=$ $(1,0,0), P_{2}:=(0,1,0)$ and $P_{3}:=(0,0,1)$, and each tetrahedron $K \in \mathcal{T}_{h}$ can be obtained from $\hat{K}$ by means of an invertible affine map $F_{K}(\hat{\mathbf{x}})=B_{K} \hat{\mathbf{x}}+b_{K}$.

Let us denote the local interpolation operator by $\Pi_{K}^{k}$. The relation $\Pi_{K}^{k}\left(\mathbf{v}_{\mid K}\right)=$ $\left(\Pi_{h}^{k} \mathbf{v}\right)_{\mid K}$ clearly holds. Moreover, as in Nédélec [12], consider the map

$$
\hat{\mathbf{v}}=B_{K}^{T} \mathbf{v} \circ F_{K}
$$

which easily yields $\left(\Pi_{K}^{k} \mathbf{v}\right)^{\wedge}=\Pi_{\hat{K}}^{k} \hat{\mathbf{v}}$.

Finally, for the sake of convenience we introduce the matrix

$$
\operatorname{Rot} \mathbf{v}:=\left(\begin{array}{ccc}
0 & \frac{\partial v_{2}}{\partial x_{1}}-\frac{\partial v_{1}}{\partial x_{2}} & \frac{\partial v_{3}}{\partial x_{1}}-\frac{\partial v_{1}}{\partial x_{3}} \\
\frac{\partial v_{1}}{\partial x_{2}}-\frac{\partial v_{2}}{\partial x_{1}} & 0 & \frac{\partial v_{3}}{\partial x_{2}}-\frac{\partial v_{2}}{\partial x_{3}} \\
\frac{\partial v_{1}}{\partial x_{3}}-\frac{\partial v_{3}}{\partial x_{1}} & \frac{\partial v_{2}}{\partial x_{3}}-\frac{\partial v_{3}}{\partial x_{2}} & 0
\end{array}\right),
$$


in such a way that

$$
\operatorname{Rot} \mathbf{v}(\mathbf{x})=\left(B_{K}^{T}\right)^{-1} \operatorname{Rot} \hat{\mathbf{v}}\left(F_{K}^{-1}(\mathbf{x})\right) B_{K}^{-1}
$$

We have

Lemma 5.2. Let $\mathcal{T}_{h}$ be a regular family of triangulations. Then there exists a constant $C>0$ such that

$$
\begin{aligned}
\|\mathbf{v}\|_{0, K}^{2} & \leq C h_{K}\|\hat{\mathbf{v}}\|_{0, \hat{K}}^{2} \\
\|\operatorname{rot} \mathbf{v}\|_{0, K}^{2} & \leq C h_{K}^{-1}\|\operatorname{rot} \hat{\mathbf{v}}\|_{0, \hat{K}}^{2}
\end{aligned}
$$

for each $\mathbf{v} \in H(\operatorname{rot} ; K)$ and $K \in \mathcal{T}_{h}$.

Proof. The procedure is classic, and we report it here for the sake of completeness. Firstly we have

$$
\begin{aligned}
\|\mathbf{v}\|_{0, K}^{2} & =\int_{K}|\mathbf{v}(\mathbf{x})|^{2} d \mathbf{x}=\int_{K}\left|\left(B_{K}^{T}\right)^{-1} \hat{\mathbf{v}}\left(F_{K}^{-1}(\mathbf{x})\right)\right|^{2} d \mathbf{x} \\
& =\left|\operatorname{det} B_{K}\right| \int_{\hat{K}}\left|\left(B_{K}^{T}\right)^{-1} \hat{\mathbf{v}}(\hat{\mathbf{x}})\right|^{2} d \hat{\mathbf{x}} \\
& \leq\left|\operatorname{det} B_{K}\right|\left\|\left(B_{K}^{T}\right)^{-1}\right\|^{2}\|\hat{\mathbf{v}}\|_{0, \hat{K}}^{2}
\end{aligned}
$$

Analogously,

$$
\begin{aligned}
\|\operatorname{rot} \mathbf{v}\|_{0, K}^{2} & =\frac{1}{2} \int_{K}|\operatorname{Rot} \mathbf{v}(\mathbf{x})|^{2} d \mathbf{x}=\frac{1}{2} \int_{K}\left|\left(B_{K}^{T}\right)^{-1} \operatorname{Rot} \hat{\mathbf{v}}\left(F_{K}^{-1}(\mathbf{x})\right) B_{K}^{-1}\right|^{2} d \mathbf{x} \\
& =\frac{1}{2}\left|\operatorname{det} B_{K}\right| \int_{\hat{K}}\left|\left(B_{K}^{T}\right)^{-1} \operatorname{Rot} \hat{\mathbf{v}}(\hat{\mathbf{x}}) B_{K}^{-1}\right|^{2} d \hat{\mathbf{x}} \\
& \leq C\left|\operatorname{det} B_{K}\right|\left\|\left(B_{K}^{T}\right)^{-1}\right\|^{2}\left\|B_{K}^{-1}\right\|^{2}\|\operatorname{rot} \hat{\mathbf{v}}\|_{0, \hat{K}}^{2} .
\end{aligned}
$$

The proof then follows by noticing that $\left\|B_{K}^{-1}\right\| \leq C h_{K}^{-1}$ and $\left|\operatorname{det} B_{K}\right| \leq C h_{K}^{3}$.

Using this Lemma we find

$$
\left\|\mathbf{v}-\Pi_{K}^{k} \mathbf{v}\right\|_{H(\operatorname{rot} ; K)}^{2} \leq C\left(h_{K}\left\|\hat{\mathbf{v}}-\Pi_{\hat{K}}^{k} \hat{\mathbf{v}}\right\|_{0, \hat{K}}^{2}+h_{K}^{-1}\left\|\operatorname{rot}\left(\hat{\mathbf{v}}-\Pi_{\hat{K}}^{k} \hat{\mathbf{v}}\right)\right\|_{0, \hat{K}}^{2}\right) .
$$

Now we want to write the term $\operatorname{rot}\left(\Pi_{\hat{K}}^{k} \hat{\mathbf{v}}\right)$ in an equivalent form.

To start with, let us consider the case $k \geq 2$. As in Nédélec [13], for $l \geq 1$ introduce the finite element space

$$
M_{h}^{l}:=\left\{\mathbf{v}_{h} \in H(\operatorname{div} ; \Omega) \mid \mathbf{v}_{h \mid K} \in\left(\mathbf{P}_{l}\right)^{3} \forall K \in \mathcal{T}_{h}\right\}
$$

with the moments

$$
\tilde{m}_{1}(\mathbf{v}):=\left\{\int_{f}(\mathbf{v} \cdot \mathbf{n}) q \text { for all } q \in \mathbf{P}_{l}(f) \text { for the four faces } f \text { of } K\right\},
$$

at which one has to add, in the case $l \geq 2$, also

$$
\tilde{m}_{2}(\mathbf{v}):=\left\{\int_{K} \mathbf{v} \cdot \mathbf{q} \text { for all } q \mathbf{q} \in R_{l-1}(K)\right\} .
$$

These moments are "div-conforming" and determine a unique element of $\left(\mathbf{P}_{l}\right)^{3}$. We will denote by $\pi_{h}^{l}$ the interpolation operator related to $M_{h}^{l}$, which is clearly well defined and continuous in $\left(H^{r}(\Omega)\right)^{3}, r>1 / 2$, and by $\pi_{K}^{l}$ the local interpolation operator. Again, we have $\pi_{K}^{l}\left(\mathbf{v}_{\mid K}\right)=\left(\pi_{h}^{l} \mathbf{v}\right)_{\mid K}$.

The following lemma was proved by Nédélec in [13], Proposition 2. 
Lemma 5.3. For each function $\hat{\mathbf{v}} \in H^{r}(\operatorname{rot} ; \hat{K}), r>1 / 2$, and for each $k \geq 2$ the following relation

$$
\operatorname{rot}\left(\Pi_{\hat{K}}^{k} \hat{\mathbf{v}}\right)=\pi_{\hat{K}}^{k-1}(\operatorname{rot} \hat{\mathbf{v}})
$$

holds.

Notice that the proof in [13] refers to the curl-conforming Nédélec elements of second type; however, the curl of the interpolant is the same for both types of Nédélec elements. Notice also that (though not explicitly underlined) in [13], Proposition 2 , it is assumed that $k-1 \geq 1$, i.e., $k \geq 2$. Finally, there the result is stated for $\hat{\mathbf{v}} \in H^{2}(\hat{K})$, but this assumption can be weakened, as a consequence of Lemma 5.1.

Consider now the case $k=1$. We denote by $f_{i}$ the face of $\hat{K}$ orthogonal to the axis $x_{i}, i=1,2,3$. The following operator $\pi_{\hat{K}}^{0}:\left(H^{r}(\hat{K})\right)^{3} \rightarrow \mathbf{R}^{3}$,

$$
\pi_{\hat{K}}^{0} \hat{\mathbf{v}}=-2\left(\begin{array}{c}
\int_{f_{1}} \hat{\mathbf{v}} \cdot \mathbf{n} \\
\int_{f_{2}} \hat{\mathbf{v}} \cdot \mathbf{n} \\
\int_{f_{3}} \hat{\mathbf{v}} \cdot \mathbf{n}
\end{array}\right),
$$

is clearly well defined and continuous for each $r>1 / 2$. Moreover

Lemma 5.4. For each function $\hat{\mathbf{v}} \in H^{r}(\operatorname{rot} ; \hat{K}), r>1 / 2$, the following relation

$$
\operatorname{rot}\left(\Pi_{\hat{K}}^{1} \hat{\mathbf{v}}\right)=\pi_{\hat{K}}^{0}(\operatorname{rot} \hat{\mathbf{v}})
$$

holds.

Proof. As $k=1$ we only have to deal with the moments of the first type $\hat{m}_{1}(\hat{\mathbf{v}})=$ $\int_{a} \hat{\mathbf{v}} \cdot \mathbf{t}_{a}$. Denote by $\hat{m}^{s}(\hat{\mathbf{v}})$ the degree of freedom on $\hat{K}$ associated to the edge $a_{s}$, $s=1, \ldots, 6$, where $a_{1}=P_{0} P_{1}, a_{2}=P_{0} P_{2}, a_{3}=P_{0} P_{3}, a_{4}=P_{3} P_{2}, a_{5}=P_{1} P_{3}$ and $a_{6}=P_{2} P_{1}$. It can be easily shown that the basis $\hat{\boldsymbol{\psi}}_{s}$ of $R_{1}$ on $\hat{K}$ satisfying $\hat{m}^{s}\left(\hat{\boldsymbol{\psi}}_{j}\right)=\delta_{s j}$ is given by

$$
\begin{gathered}
\hat{\boldsymbol{\psi}}_{1}=\left(\begin{array}{c}
1-\hat{y}-\hat{z} \\
\hat{x} \\
\hat{x}
\end{array}\right), \hat{\boldsymbol{\psi}}_{2}=\left(\begin{array}{c}
\hat{y} \\
1-\hat{x}-\hat{z} \\
\hat{y}
\end{array}\right), \hat{\boldsymbol{\psi}}_{3}=\left(\begin{array}{c}
\hat{z} \\
\hat{z} \\
1-\hat{x}-\hat{y}
\end{array}\right), \\
\hat{\boldsymbol{\psi}}_{4}=\left(\begin{array}{c}
0 \\
\hat{z} \\
-\hat{y}
\end{array}\right), \hat{\boldsymbol{\psi}}_{5}=\left(\begin{array}{c}
-\hat{z} \\
0 \\
\hat{x}
\end{array}\right), \hat{\boldsymbol{\psi}}_{6}=\left(\begin{array}{c}
\hat{y} \\
-\hat{x} \\
0
\end{array}\right) .
\end{gathered}
$$

Let us notice that

$$
\begin{aligned}
& \operatorname{rot} \hat{\boldsymbol{\psi}}_{1}=\left(\begin{array}{c}
0 \\
-2 \\
2
\end{array}\right), \operatorname{rot} \hat{\boldsymbol{\psi}}_{2}=\left(\begin{array}{c}
2 \\
0 \\
-2
\end{array}\right), \operatorname{rot} \hat{\boldsymbol{\psi}}_{3}=\left(\begin{array}{c}
-2 \\
2 \\
0
\end{array}\right), \\
& \operatorname{rot} \hat{\boldsymbol{\psi}}_{4}=\left(\begin{array}{c}
-2 \\
0 \\
0
\end{array}\right), \operatorname{rot} \hat{\boldsymbol{\psi}}_{5}=\left(\begin{array}{c}
0 \\
-2 \\
0
\end{array}\right), \operatorname{rot} \hat{\boldsymbol{\psi}}_{6}=\left(\begin{array}{c}
0 \\
0 \\
-2
\end{array}\right),
\end{aligned}
$$


and therefore

$$
\operatorname{rot}\left(\Pi_{\hat{K}}^{1} \hat{\mathbf{v}}\right)=2\left(\begin{array}{c}
\hat{m}^{2}(\hat{\mathbf{v}})-\hat{m}^{3}(\hat{\mathbf{v}})-\hat{m}^{4}(\hat{\mathbf{v}}) \\
-\hat{m}^{1}(\hat{\mathbf{v}})+\hat{m}^{3}(\hat{\mathbf{v}})-\hat{m}^{5}(\hat{\mathbf{v}}) \\
\hat{m}^{1}(\hat{\mathbf{v}})-\hat{m}^{2}(\hat{\mathbf{v}})-\hat{m}^{6}(\hat{\mathbf{v}})
\end{array}\right) .
$$

Taking the unit vector $\mathbf{t}=\mathbf{n} \times \boldsymbol{\nu}$ as in Lemma 5.1, it follows that

$$
\operatorname{rot}\left(\Pi_{\hat{K}}^{1} \hat{\mathbf{v}}\right)=-2\left(\begin{array}{c}
\int_{\partial f_{1}} \hat{\mathbf{v}} \cdot \mathbf{t}_{1} \\
\int_{\partial f_{2}} \hat{\mathbf{v}} \cdot \mathbf{t}_{2} \\
\int_{\partial f_{3}} \hat{\mathbf{v}} \cdot \mathbf{t}_{3}
\end{array}\right)=-2\left(\begin{array}{c}
\int_{f_{1}} \operatorname{rot} \hat{\mathbf{v}} \cdot \mathbf{n} \\
\int_{f_{2}} \operatorname{rot} \hat{\mathbf{v}} \cdot \mathbf{n} \\
\int_{f_{3}} \operatorname{rot} \hat{\mathbf{v}} \cdot \mathbf{n}
\end{array}\right)=\pi_{\hat{K}}^{0}(\operatorname{rot} \hat{\mathbf{v}}),
$$

having used the Stokes Theorem on each face $f_{i}$.

We are now in a position to consider the operators

$$
I-\Pi_{\hat{K}}^{k}: H^{r}(\operatorname{rot}, \hat{K}) \rightarrow\left(L^{2}(\hat{K})\right)^{3}, \quad I-\pi_{\hat{K}}^{k-1}:\left(H^{r}(\hat{K})\right)^{3} \rightarrow\left(L^{2}(\hat{K})\right)^{3},
$$

which are clearly linear and continuous. Since we are dealing with a non-integer $r$, with $1 / 2<r<k$, it follows that the integral part $[r]$ of $r$ satisfies $[r] \leq k-1$. Hence, the operators above take value zero for each polynomial in $\mathbf{P}_{[r]}$, and applying the Bramble-Hilbert Lemma we find

$$
\begin{gathered}
\left\|\left(I-\Pi_{\hat{K}}^{k}\right) \hat{\mathbf{v}}\right\|_{0, \hat{K}}^{2} \leq C \inf _{\hat{\mathbf{p}} \in\left(\mathbf{P}_{[r]}\right)^{3}}\|\hat{\mathbf{v}}+\hat{\mathbf{p}}\|_{H^{r}(\operatorname{rot} ; \hat{K})}^{2}, \\
\left\|\left(I-\pi_{\hat{K}}^{k-1}\right) \operatorname{rot} \hat{\mathbf{v}}\right\|_{0, \hat{K}}^{2} \leq C \inf _{\hat{\mathbf{p}} \in\left(\mathbf{P}_{[r]}\right)^{3}}\|\operatorname{rot} \hat{\mathbf{v}}+\hat{\mathbf{p}}\|_{r, \hat{K}}^{2} .
\end{gathered}
$$

By repeating the proof of the Deny-Lions Lemma we finally have

$$
\begin{gathered}
\inf _{\hat{\mathbf{p}} \in\left(\mathbf{P}_{[r]}\right)^{3}}\|\hat{\mathbf{v}}+\hat{\mathbf{p}}\|_{H^{r}(\operatorname{rot} ; \hat{K})}^{2} \leq C\left(|\hat{\mathbf{v}}|_{r, \hat{K}}^{2}+|\operatorname{rot} \hat{\mathbf{v}}|_{[r], \hat{K}}^{2}+|\operatorname{rot} \hat{\mathbf{v}}|_{r, \hat{K}}^{2}\right), \\
\inf _{\hat{\mathbf{p}} \in\left(\mathbf{P}_{[r])^{3}}\right.} \| \operatorname{rot} \hat{\mathbf{v}}+\left.\hat{\mathbf{p}}\right|_{r, \hat{K}} ^{2} \leq C|\operatorname{rot} \hat{\mathbf{v}}|_{r, \hat{K}}^{2} .
\end{gathered}
$$

We recall that for an integer $k$ the semi-norm in $\left(H^{k}(\Omega)\right)^{3}$ is defined as

$$
|\hat{\mathbf{v}}|_{k, \hat{K}}:=\left(\sum_{|\alpha|=k}\left\|D^{\alpha} \hat{\mathbf{v}}\right\|_{0, \hat{K}}^{2}\right)^{1 / 2}
$$

whereas for a non-integer value $s$ it holds

$$
|\hat{\mathbf{v}}|_{s, \hat{K}}:=\left(\sum_{|\alpha|=[s]}\left|D^{\alpha} \hat{\mathbf{v}}\right|_{s-[s], \hat{K}}^{2}\right)^{1 / 2}
$$

where for $\theta \in(0,1)$ we have set

$$
|\hat{\mathbf{v}}|_{\theta, \hat{K}}:=\left(\int_{\hat{K}} \int_{\hat{K}} \frac{|\hat{\mathbf{v}}(\hat{\mathbf{x}})-\hat{\mathbf{v}}(\hat{\mathbf{y}})|^{2}}{|\hat{\mathbf{x}}-\hat{\mathbf{y}}|^{3+2 \theta}} d \hat{\mathbf{x}} d \hat{\mathbf{y}}\right)^{1 / 2} .
$$


From (5.9)-(5.13) we have thus obtained

$$
\begin{aligned}
\left\|\mathbf{v}-\Pi_{K}^{k} \mathbf{v}\right\|_{H(\operatorname{rot} ; K)} & \leq C\left(h_{K}\left\|\hat{\mathbf{v}}-\Pi_{\hat{K}}^{k} \hat{\mathbf{v}}\right\|_{0, \hat{K}}^{2}+h_{K}^{-1}\left\|\operatorname{rot} \hat{\mathbf{v}}-\pi_{\hat{K}}^{k-1}(\operatorname{rot} \hat{\mathbf{v}})\right\|_{0, \hat{K}}^{2}\right) \\
& \leq C\left[h_{K}\left(|\hat{\mathbf{v}}|_{r, \hat{K}}^{2}+|\operatorname{rot} \mathbf{v}|_{[r], \hat{K}}^{2}+|\operatorname{rot} \hat{\mathbf{v}}|_{r, \hat{K}}^{2}\right)+h_{K}^{-1}|\operatorname{rot} \hat{\mathbf{v}}|_{r, \hat{K}}^{2}\right] \\
& \leq C\left[h_{K}\left(|\hat{\mathbf{v}}|_{r, \hat{K}}^{2}+|\operatorname{rot} \hat{\mathbf{v}}|_{[r], \hat{K}}^{2}\right)+h_{K}^{-1}|\operatorname{rot} \hat{\mathbf{v}}|_{r, \hat{K}}^{2}\right] .
\end{aligned}
$$

We need the following result.

Lemma 5.5. Let $\mathcal{T}_{h}$ be a regular family of triangulations. Then there exists a constant $C>0$ such that

$$
\begin{aligned}
|\hat{\mathbf{v}}|_{s, \hat{K}}^{2} & \leq C h_{K}^{-1+2 s}|\mathbf{v}|_{s, K}^{2} \\
|\operatorname{rot} \hat{\mathbf{v}}|_{s, \hat{K}}^{2} & \leq C h_{K}^{1+2 s}|\operatorname{rot} \mathbf{v}|_{s, K}^{2}
\end{aligned}
$$

for each real number $s \geq 0$.

Proof. We will present the proof only for $0<s<1$, the other cases being standard. We have

$$
\begin{aligned}
|\hat{\mathbf{v}}|_{s, \hat{K}}^{2} & =\int_{\hat{K}} \int_{\hat{K}} \frac{|\hat{\mathbf{v}}(\hat{\mathbf{x}})-\hat{\mathbf{v}}(\hat{\mathbf{y}})|^{2}}{|\hat{\mathbf{x}}-\hat{\mathbf{y}}|^{3+2 s}} d \hat{\mathbf{x}} d \hat{\mathbf{y}} \\
& =\left|\operatorname{det} B_{K}\right|^{-2} \int_{K} \int_{K} \frac{\left|B_{K}^{T}(\mathbf{v}(\mathbf{x})-\mathbf{v}(\mathbf{y}))\right|^{2}}{\left|B_{K}^{-1}(\mathbf{x}-\mathbf{y})\right|^{3+2 s}} d \mathbf{x} d \mathbf{y} .
\end{aligned}
$$

We can write

$$
|\mathbf{x}-\mathbf{y}|=\left|B_{K} B_{K}^{-1}(\mathbf{x}-\mathbf{y})\right| \leq|| B_{K}||\left|B_{K}^{-1}(\mathbf{x}-\mathbf{y})\right|
$$

therefore, $\left|B_{K}^{-1}(\mathbf{x}-\mathbf{y})\right| \geq\left\|B_{K}\right\|^{-1}|\mathbf{x}-\mathbf{y}|$. Hence,

$$
\begin{aligned}
|\hat{\mathbf{v}}|_{s, \hat{K}}^{2} & \leq\left|\operatorname{det} B_{K}\right|^{-2} \| B_{K}||^{3+2 s} \int_{K} \int_{K} \frac{\left|B_{K}^{T}(\mathbf{v}(\mathbf{x})-\mathbf{v}(\mathbf{y}))\right|^{2}}{|\mathbf{x}-\mathbf{y}|^{3+2 s}} d \mathbf{x} d \mathbf{y} \\
& \leq C\left|\operatorname{det} B_{K}\right|^{-2}\left\|B_{K}\right\|^{3+2 s}|| B_{K}^{T} \|^{2}|\mathbf{v}|_{s, K}^{2} \\
& \leq C h_{K}^{-1+2 s}|\mathbf{v}|_{s, K}^{2} .
\end{aligned}
$$

In an analogous way

$$
\begin{aligned}
|\operatorname{rot} \hat{\mathbf{v}}|_{s, \hat{K}}^{2} & =\frac{1}{2} \int_{\hat{K}} \int_{\hat{K}} \frac{|\operatorname{Rot} \hat{\mathbf{v}}(\hat{\mathbf{x}})-\operatorname{Rot} \hat{\mathbf{v}}(\hat{\mathbf{y}})|^{2}}{|\hat{\mathbf{x}}-\hat{\mathbf{y}}|^{3+2 s}} d \hat{\mathbf{x}} d \hat{\mathbf{y}} \\
& =\frac{1}{2}\left|\operatorname{det} B_{K}\right|^{-2} \int_{K} \int_{K} \frac{\left|B_{K}^{T}(\operatorname{Rot} \mathbf{v}(\mathbf{x})-\operatorname{Rot} \mathbf{v}(\mathbf{y})) B_{K}\right|^{2}}{\left|B_{K}^{-1}(\mathbf{x}-\mathbf{y})\right|^{3+2 s}} d \mathbf{x} d \mathbf{y} \\
& \leq\left.\left. C\left|\operatorname{det} B_{K}\right|^{-2}|| B_{K}\right|^{5+2 s}|| B_{K}^{T}\right|^{2}|\operatorname{rot} \mathbf{v}|_{s, K}^{2} \\
& \leq C h_{K}^{1+2 s}|\operatorname{rot} \mathbf{v}|_{s, K}^{2},
\end{aligned}
$$

which concludes the proof.

We can conclude with the following interpolation result.

Proposition 5.6. Let $r$ be a non-integer with $1 / 2<r<k$. Let $\mathcal{T}_{h}$ be a regular family of triangulations. Then there exists a constant $C>0$, independent of $h$, such that

$$
\left\|\mathbf{v}-\Pi_{h}^{k} \mathbf{v}\right\|_{H(\operatorname{rot} ; \Omega)} \leq C h^{r}\|\mathbf{v}\|_{H^{r}(\operatorname{rot} ; \Omega)}
$$

for each $\mathbf{v} \in H^{r}(\operatorname{rot} ; \Omega)$. 
Proof. Using the additivity of the integral we can write

$$
\begin{aligned}
\left\|\mathbf{v}-\Pi_{h}^{k} \mathbf{v}\right\|_{H(\operatorname{rot} ; \Omega)}^{2} & =\sum_{K \in \mathcal{T}_{h}}\left(\left\|\mathbf{v}-\Pi_{K}^{k} \mathbf{v}\right\|_{0, K}^{2}+\left\|\operatorname{rot} \mathbf{v}-\pi_{K}^{k-1} \operatorname{rot} \mathbf{v}\right\|_{0, K}^{2}\right) \\
& \leq \sum_{K \in \mathcal{T}_{h}}\left[h_{K}\left(|\hat{\mathbf{v}}|_{r, \hat{K}}^{2}+|\operatorname{rot} \hat{\mathbf{v}}|_{[r], \hat{K}}^{2}\right)+h_{K}^{-1}|\operatorname{rot} \hat{\mathbf{v}}|_{r, \hat{K}}^{2}\right] .
\end{aligned}
$$

From Lemma 5.5 we find

$$
\begin{aligned}
\left\|\mathbf{v}-\Pi_{h}^{k} \mathbf{v}\right\|_{H(\operatorname{rot} ; \Omega)}^{2} \leq & \sum_{K \in \mathcal{T}_{h}}\left[h_{K}\left(h_{K}^{-1+2 r}|\mathbf{v}|_{r, K}^{2}+h_{K}^{1+2[r]}|\operatorname{rot} \mathbf{v}|_{[r], K}^{2}\right)\right. \\
& \left.\quad+h_{K}^{-1} h_{K}^{1+2 r}|\operatorname{rot} \mathbf{v}|_{r, K}^{2}\right] \\
\leq & C \sum_{K \in \mathcal{T}_{h}}\left(h_{K}^{2 r}|\mathbf{v}|_{r, K}^{2}+h_{K}^{2+2[r]}|\operatorname{rot} \mathbf{v}|_{[r], K}^{2}+h_{K}^{2 r}|\operatorname{rot} \mathbf{v}|_{r, K}^{2}\right) \\
\leq & C h^{2 r}\|\mathbf{v}\|_{H^{r}(\operatorname{rot} ; \Omega)}^{2},
\end{aligned}
$$

and the thesis is proved.

The estimates (5.7), (5.8) and (5.14) yield the interpolation estimate (5.6), and we are now in a position to conclude the proof of Theorem B.

Proof of Theorem B. We are going to follow the lines of the proof of Céa Lemma. We have

$$
\begin{aligned}
\| \mathbf{F}_{1, \Gamma} \gamma_{h} & -\mathbf{F}_{1, \Gamma}^{h} \boldsymbol{\gamma}_{h} \|_{H\left(\mathrm{rot} ; \Omega_{1}\right)}^{2}=\left(\left(\mathbf{F}_{1, \Gamma} \boldsymbol{\gamma}_{h}-\mathbf{F}_{1, \Gamma}^{h} \boldsymbol{\gamma}_{h}, \mathbf{F}_{1, \Gamma} \boldsymbol{\gamma}_{h}-\mathbf{F}_{1, \Gamma}^{h} \boldsymbol{\gamma}_{h}\right)\right)_{\Omega_{1}} \\
& =\left(\left(\mathbf{F}_{1, \Gamma} \boldsymbol{\gamma}_{h}-\mathbf{F}_{1, \Gamma}^{h} \boldsymbol{\gamma}_{h}, \mathbf{F}_{1, \Gamma} \boldsymbol{\gamma}_{h}-\Pi_{h}^{k} \mathbf{F}_{1, \Gamma} \boldsymbol{\gamma}_{h}+\Pi_{h}^{k} \mathbf{F}_{1, \Gamma} \boldsymbol{\gamma}_{h}-\mathbf{F}_{1, \Gamma}^{h} \boldsymbol{\gamma}_{h}\right)\right)_{\Omega_{1}} .
\end{aligned}
$$

Let us take now $\gamma_{h} \in \mathcal{X}_{\Gamma, h}$. A basis $\phi_{1, j}$ in $\mathcal{X}_{\Gamma, h}$ is given by $\left(\mathbf{n}_{\Gamma} \times \boldsymbol{\psi}_{1, j}\right)_{\mid \Gamma}$, where $\boldsymbol{\psi}_{1, j}$ are basis functions of $V_{1, h}$. Moreover, due to relation (5.5), for any tangential element $\gamma \in\left(H^{r-1 / 2}(\Gamma)\right)^{3} \cap H\left(\operatorname{div}_{\tau} ; \Gamma\right)$ the degrees of freedom related to $\mathcal{X}_{\Gamma, h}$ are given by $-\int_{a} \boldsymbol{\gamma} \cdot \boldsymbol{\nu} q$ for each $q \in \mathbf{P}_{k-1}(a)$ and by $-\int_{f} \boldsymbol{\gamma} \cdot \mathbf{q}$ for each $\mathbf{q} \in\left(\mathbf{P}_{k-2}(f)\right)^{2}$, where $a$ and $f$ are any edge and face of $\Gamma$, respectively. Therefore, for any $\mathbf{v}_{1} \in$ $H^{r}\left(\operatorname{rot} ; \Omega_{1}\right)$ the interpolant on $\Gamma$ of $\left(\mathbf{n}_{\Gamma} \times \mathbf{v}_{1}\right)_{\mid \Gamma}$ is given by $\left(\mathbf{n}_{\Gamma} \times \Pi_{h}^{k} \mathbf{v}_{1}\right)_{\mid \Gamma}$; hence, $\left(\mathbf{n}_{\Gamma} \times \Pi_{h}^{k} \mathbf{F}_{1, \Gamma} \gamma_{h}\right)_{\mid \Gamma}=\gamma_{h}$. Consequently, we have $\left(\Pi_{h}^{k} \mathbf{F}_{1, \Gamma} \boldsymbol{\gamma}_{h}-\mathbf{F}_{1, \Gamma}^{h} \gamma_{h}\right) \in V_{1, h}^{0}$ and

$$
\left(\left(\mathbf{F}_{1, \Gamma} \boldsymbol{\gamma}_{h}-\mathbf{F}_{1, \Gamma}^{h} \boldsymbol{\gamma}_{h}, \Pi_{h}^{k} \mathbf{F}_{1, \Gamma} \boldsymbol{\gamma}_{h}-\mathbf{F}_{1, \Gamma}^{h} \boldsymbol{\gamma}_{h}\right)\right)_{\Omega_{1}}=0 .
$$

Hence we find

$$
\left\|\mathbf{F}_{1, \Gamma} \boldsymbol{\gamma}_{h}-\mathbf{F}_{1, \Gamma}^{h} \boldsymbol{\gamma}_{h}\right\|_{H\left(\mathrm{rot} ; \Omega_{1}\right)} \leq\left\|\mathbf{F}_{1, \Gamma} \boldsymbol{\gamma}_{h}-\Pi_{h}^{k} \mathbf{F}_{1, \Gamma} \boldsymbol{\gamma}_{h}\right\|_{H\left(\mathrm{rot} ; \Omega_{1}\right)} .
$$

From the assumption $\mathbf{F}_{1, \Gamma} \gamma_{h} \in H^{r}\left(\operatorname{rot} ; \Omega_{1}\right)$ and the interpolation inequality (5.6) we finally find

$$
\left\|\mathbf{F}_{1, \Gamma} \gamma_{h}-\mathbf{F}_{1, \Gamma}^{h} \gamma_{h}\right\|_{H\left(\operatorname{rot} ; \Omega_{1}\right)} \leq K_{2} h^{r}\left\|\mathbf{F}_{1, \Gamma} \gamma_{h}\right\|_{H^{r}\left(\operatorname{rot} ; \Omega_{1}\right)},
$$

and Theorem B is completely proved. 


\section{Proof of Theorem C}

We have to prove that if $\mathcal{M}_{h}$ is a quasi-uniform family of triangulations of $\partial \Omega$, then there exists a constant $C>0$ such that for each $\boldsymbol{\eta}_{h} \in \mathcal{X}_{\partial \Omega, h}$ it holds

$$
\left\|\boldsymbol{\eta}_{h}\right\|_{\epsilon, \partial \Omega}+\left\|\operatorname{div}_{\tau} \boldsymbol{\eta}_{h}\right\|_{\epsilon, \partial \Omega} \leq C h^{-\frac{1}{2}-\epsilon}\left(\left\|\boldsymbol{\eta}_{h}\right\|_{-1 / 2, \partial \Omega}+\left\|\operatorname{div}_{\tau} \boldsymbol{\eta}_{h}\right\|_{-1 / 2, \partial \Omega}\right) .
$$

Noticing that both $\boldsymbol{\eta}_{h}$ and $\operatorname{div}_{\tau} \boldsymbol{\eta}_{h}$ are polynomials in each triangle on $\partial \Omega$, one can apply the inverse inequality for the non-integer exponent $\epsilon$ obtaining

$$
\left\|\boldsymbol{\eta}_{h}\right\|_{\epsilon, \partial \Omega}+\left\|\operatorname{div}_{\tau} \boldsymbol{\eta}_{h}\right\|_{\epsilon, \partial \Omega} \leq C h^{-\epsilon}\left(\left\|\boldsymbol{\eta}_{h}\right\|_{0, \partial \Omega}+\left\|\operatorname{div}_{\tau} \boldsymbol{\eta}_{h}\right\|_{0, \partial \Omega}\right) .
$$

It remains for us to show that for each real scalar function $z_{h} \in L^{2}(\partial \Omega)$ and such that $z_{h \mid T} \in \mathbf{P}_{k}$ for each triangle $T \in \mathcal{M}_{h}$ we have

$$
\left\|z_{h}\right\|_{0, \partial \Omega} \leq C h^{-1 / 2}\left\|z_{h}\right\|_{-1 / 2, \partial \Omega} .
$$

A similar result in the two-dimensional case can be found in Quarteroni, Sacchi Landriani and Valli [14]. We are going to adapt their proof to the case under consideration. Let us set $M_{h}:=\left\{v \in H^{1}(\partial \Omega) \mid v_{\mid T} \in \mathbf{P}_{k+3} \forall T \in \mathcal{M}_{h}\right\}$. For each $\phi \in L^{2}(\partial \Omega)$ denote by $\phi_{h}^{*}$ the $L^{2}(\partial \Omega)$-orthogonal projection of $\phi$ onto $M_{h}$. Moreover, denote by $\phi_{h}^{* *}$ the function belonging to $M_{h}$ which is defined as

$$
\phi_{h \mid T}^{* *}=\phi_{h \mid T}^{*}+\sum_{|\alpha| \leq k}\left(\phi-\phi_{h}^{*},[\mathbf{M}(\mathbf{x})]^{\alpha}\right)_{T} P_{\alpha, T},
$$

where $\mathbf{M}(\mathbf{x})$ is the rigid motion sending $T$ on the $(x, y)$-plane with one vertex in $(0,0)$, and $P_{\alpha, T} \in \mathbf{P}_{k+1}$ satisfies $P_{\alpha, T \mid \partial T}=0$ and

$$
\left(P_{\alpha, T},[\mathbf{M}(\mathbf{x})]^{\beta}\right)_{T}=\left\{\begin{array}{ll}
0 & \text { if } \alpha \neq \beta \\
1 & \text { if } \alpha=\beta,
\end{array} \quad|\alpha|,|\beta| \leq k .\right.
$$

Clearly, the function $z_{h \mid T}$ can be written as a linear combination of $[\mathbf{M}(\mathbf{x})]^{\alpha},|\alpha| \leq k$; therefore, it follows at once that $\int_{\Omega} z_{h} \phi=\int_{\Omega} z_{h} \phi_{h}^{* *}$ for each $\phi \in L^{2}(\Omega)$, and we have

$$
\begin{aligned}
\left\|z_{h}\right\|_{0, \partial \Omega} & =\sup _{\substack{\phi \in L^{2}(\partial \Omega) \\
\phi \neq 0}} \frac{\int_{\partial \Omega} z_{h} \phi}{\|\phi\|_{0, \partial \Omega}}=\sup _{\substack{\phi \in L^{2}(\partial \Omega) \\
\phi \neq 0}} \frac{\int_{\partial \Omega} z_{h} \phi_{h}^{* *}}{\|\phi\|_{0, \partial \Omega}} \\
& \leq \sup _{\substack{\phi \in L^{2}(\partial \Omega) \\
\phi \neq 0}} \frac{\left\|z_{h}\right\|_{-1 / 2, \partial \Omega}\left\|\phi_{h}^{* *}\right\|_{1 / 2, \partial \Omega}}{\|\phi\|_{0, \partial \Omega}} .
\end{aligned}
$$

By using the inverse inequality as in (6.2) we obtain

$$
\left\|\phi_{h}^{* *}\right\|_{1 / 2, \partial \Omega} \leq C h^{-1 / 2}\left\|\phi_{h}^{* *}\right\|_{0, \partial \Omega}
$$

hence,

$$
\left\|z_{h}\right\|_{0, \partial \Omega} \leq C h^{-1 / 2}\left\|z_{h}\right\|_{-1 / 2, \partial \Omega} \sup _{\substack{\phi \in L^{2}(\partial \Omega) \\ \phi \neq 0}} \frac{\left\|\phi_{h}^{* *}\right\|_{0, \partial \Omega}}{\|\phi\|_{0, \partial \Omega}} .
$$

To conclude the proof, we have to show that

$$
\left\|\phi_{h}^{* *}\right\|_{0, \partial \Omega} \leq C\|\phi\|_{0, \partial \Omega} .
$$


We have

$$
\begin{aligned}
\left\|\phi_{h}^{* *}\right\|_{0, \partial \Omega}^{2} & =\sum_{T \in \mathcal{M}_{h}}\left\|\phi_{h}^{* *}\right\|_{0, T}^{2}=\sum_{T \in \mathcal{M}_{h}} \int_{T}\left(\phi_{h}^{*}+\sum_{|\alpha| \leq k}\left(\phi-\phi_{h}^{*},[\mathbf{M}(\mathbf{x})]^{\alpha}\right)_{T} P_{\alpha, T}\right)^{2} \\
& \leq C \sum_{T \in \mathcal{M}_{h}} \int_{T}\left(\left|\phi_{h}^{*}\right|^{2}+\sum_{|\alpha| \leq k}\left(\phi-\phi_{h}^{*},[\mathbf{M}(\mathbf{x})]^{\alpha}\right)_{T}^{2} P_{\alpha, T}^{2}\right) \\
& \leq C\left[\left\|\phi_{h}^{*}\right\|_{0, \partial \Omega}^{2}+\sum_{T \in \mathcal{M}_{h}}\left(\left\|\phi-\phi_{h}^{*}\right\|_{0, T}^{2} \sum_{|\alpha| \leq k} \int_{T}[\mathbf{M}(\mathbf{x})]^{2 \alpha} \int_{T} P_{\alpha, T}^{2}\right)\right]
\end{aligned}
$$

By a straightforward computation it can be shown that

$$
\max _{T \in \mathcal{M}_{h}} \int_{T}[\mathbf{M}(\mathbf{x})]^{2 \alpha} \int_{T} P_{\alpha, T}^{2} \leq C,
$$

uniformly with respect to $h$, and (6.5) follows by recalling that $\phi_{h}^{*}$ is the $L^{2}(\partial \Omega)$ orthogonal projection of $\phi$ onto $M_{h}$.

\section{REFERENCES}

1. R.A. Adams, Sobolev Spaces, Academic Press, New York, 1975. MR 56:9247

2. A. Alonso and A. Valli, Some remarks on the characterization of the space of tangential traces of $H($ rot; $\Omega)$ and the construction of an extension operator, Manuscr. Math. 89 (1996), 159-178. MR 96k:46057

3. A. Alonso and A. Valli, A domain decomposition approach for heterogeneous time-harmonic Maxwell equations, Comput. Meth. Appl. Mech. Engrg. 143 (1997), 97-112. MR 98b:78020

4. A. Alonso and A. Valli, Unique solvability for high-frequency heterogeneous time-harmonic Maxwell equations via the Fredholm alternative theory, Math. Meth. Appl. Sci., to appear.

5. C. Amrouche, C. Bernardi, M. Dauge and V. Girault, Vector potentials in three-dimensional nonsmooth domains, preprint R 96001, Laboratoire d'Analyse Numérique, Université Pierre et Marie Curie, Paris, 1996.

6. M. Costabel, A remark on the regularity of solutions of Maxwell's equations on Lipschitz domains, Math. Meth. Appl. Sci. 12 (1990), 365-368. MR 91c:35028

7. M. Dauge, Elliptic Boundary Value Problems on Corner Domains, Springer-Verlag, Berlin, 1988. MR 91a:35078

8. M. Křižek and P. Neittaanmäki, On time-harmonic Maxwell equations with nonhomogeneous conductivities: solvability and FE-approximation, Apl. Mat. 34 (1989), 480-499. MR 90m:35177

9. R. Leis, Exterior boundary-value problems in mathematical physics, in Trends in Applications of Pure Mathematics to Mechanics, Vol. 11, H. Zorski ed., Pitman, London, 1979, pp. 187-203. MR 81d:78016

10. P. Monk, A finite element method for approximating the time-harmonic Maxwell equations, Numer. Math. 63 (1992), 243-261. MR 94b:65134

11. P. Monk, Analysis of a finite element method for Maxwell's equations, SIAM J. Numer. Anal. 29 (1992), 714-729. MR 93k:65096

12. J.C. Nédélec, Mixed finite elements in $R^{3}$, Numer. Math. 35 (1980), 315-341. MR 81k:65125

13. J.C. Nédélec, A new family of mixed finite elements in $R^{3}$, Numer. Math. 50 (1986), 57-81. MR 88e:65145

14. A. Quarteroni, G. Sacchi Landriani and A. Valli, Coupling of viscous and inviscid Stokes equations via a domain decomposition method for finite elements, Numer. Math. 59 (1991), 831-859. MR 93b:65190

15. J. Saranen, On generalized harmonic fields in domains with anisotropic nonhomogeneous media, J. Math. Anal. Appl. 88 (1982), 104-115; Erratum: J. Math. Anal. Appl. 91 (1983), 300. MR 84d:78004a, MR 84d:78004b 
16. J. Saranen, On electric and magnetic problems for vector fields in anisotropic nonhomogeneous media, J. Math. Anal. Appl. 91 (1983), 254-275. MR 85i:78004

17. A. Valli, Orthogonal decompositions of $\left(L^{2}(\Omega)\right)^{3}$, preprint UTM 493, Dipartimento di Matematica, Università di Trento, 1996.

Dipartimento di Matematica, Università di Trento, 38050 Povo (Trento), Italy

E-mail address: alonso@science.unitn.it

E-mail address: valli@science.unitn.it 